\title{
The SUMO Conjugase Ubc9 Protects Dopaminergic Cells from Cytotoxicity and Enhances the Stability of $\alpha$-Synuclein in Parkinson's Disease Models
}

\author{
DDinesh Kumar Verma, ${ }^{1}$ Anurupa Ghosh, ${ }^{1}$ Lindsey Ruggiero, ${ }^{1}$ Etienne Cartier, ${ }^{1}$ Eric Janezic, ${ }^{1}$ \\ Dionne Williams, ${ }^{1}$ Eui-Gil Jung, ${ }^{3}$ Michael Moore, ${ }^{2}$ Jong Bok Seo, ${ }^{3}$ and ${ }^{\circ}$ Yong-Hwan Kim ${ }^{1}$
}

https://doi.org/10.1523/ENEURO.0134-20.2020

${ }^{1}$ Department of Biological Sciences/Neuroscience program, Delaware State University, Dover, DE 19901, ${ }^{2} \mathrm{Imaging}$ Core, Delaware State University, Dover, DE 19901, and ${ }^{3}$ Seoul Center, Korea Basic Science Institute, Seoul 02841, Republic of Korea

\begin{abstract}
Small ubiquitin-like modifier (SUMO) is a widespread regulatory mechanism of post-translational modification (PTM) that induces rapid and reversible changes in protein function and stability. Using SUMO conjugase Ubc9-overexpressing or knock-down cells in Parkinson's disease (PD) models, we demonstrate that SUMOylation protects dopaminergic cells against MPP + or preformed fibrils (PFFs) of $\alpha$-synuclein $(\alpha$-syn)induced toxicities in cell viability and cytotoxicity assays. In the mechanism of protection, Ubc9 overexpression significantly suppressed the MPP+ or PFF-induced reactive oxygen species (ROS) generation, while Ubc9-RNAi enhanced the toxicity-induced ROS production. Further, PFF-mediated protein aggregation was exacerbated by Ubc9-RNAi in thioflavin T staining, compared with NC1 controls. In cycloheximide (Chx)-based protein stability assays, higher protein level of $\alpha$-syn was identified in Ubc9-enhanced green fluorescent protein (EGFP) than in EGFP cells. Since there was no difference in endogenous mRNA levels of $\alpha$-syn between Ubc9 and EGFP cells in quantitative real-time PCR (qRT-PCR), we assessed the mechanisms of SUMO-mediated delayed $\alpha$-syn degradation via MG132, proteasomal inhibitor, and PMA, Iysosomal degradation inducer. Ubc9-mediated SUMOylated $\alpha$-syn avoided PMA-induced lysosomal degradation because of its high solubility. Our results suggest that Ubc9 enhances the levels of SUMO1 and ubiquitin on $\alpha$-syn and interrupts SUMO1 removal from $\alpha$-syn. In immunohistochemistry, dopaminergic axon tips in the striatum and cell bodies in the substantia nigra from Ubc9-overexpressing transgenic mice were protected from MPTP toxicities compared with wild-type (WT) siblings. Our results support that SUMOylation can be a regulatory target to protect dopaminergic neurons from oxidative stress and protein
\end{abstract}

\section{Significance Statement}

We tested whether SUMOylation enhances the solubility of aggregation-prone proteins such as $\alpha$-synuclein ( $\alpha$-syn) to prevent protein aggregation induced by oxidative stress and/or preformed fibrils (PFFs) of $\alpha$-syn. Here, we demonstrate that high levels of SUMOylation mediated by Ubc9 overexpression protect dopaminergic cells from MPTP-induced (MPP+) or PFF-induced toxicities. The protective effects are derived from the inhibition of reactive oxygen species (ROS) generation and protein aggregation. Interestingly, SUMOylated $\alpha$-syn avoided lysosomal degradation, which was not detrimental. Ubiquitin binding to lysine residues may not compete with small ubiquitin-like modifier (SUMO) binding to determine the protein half-life of $\alpha$-syn. Our findings strongly suggest that the regulation of SUMO conjugation to $\alpha$-syn can be a novel therapeutic target to prevent the formation of Lewy bodies and ROS generation. 
aggregation, with the implication that high levels of SUMOylation in dopaminergic neurons can prevent the pathologic progression of PD.

Key words: $\alpha$-synuclein; degradation; lysosome; proteostasis; SUMOylation; Ubc9

\section{Introduction}

The formation of intraneuronal inclusions called Lewy bodies, in which $\alpha$-synuclein ( $\alpha$-syn) is their major protein component, is a hallmark of Parkinson's disease (PD; Spillantini et al., 1997). Although the roles of $\alpha$-syn in neurons are not well characterized, it is known to be involved in many vital cellular functions including modulating vesicle trafficking (Pranke et al., 2011; Scott and Roy, 2012), regulating dopamine biosynthesis (Peng et al., 2005), preventing oxidation of unsaturated lipids in vesicles (Zhu et al., 2006), acting as a molecular chaperon (Chandra et al., 2005), maintaining the SNARE complex (Burré et al., 2010; Choi et al., 2013), as well as acting as a neuroprotective protein in dopaminergic cells (Jin et al., 2011). Pathologic mechanisms associated with PD have been reported that impaired proteasomal function or mutations of $\alpha$-syn (e.g., A53T) can enhance its aggregation in the striatum and substantia nigra pars compacta (SNpc; Spillantini et al., 1997; Siderowf and Stern, 2003). Among the causes of $\alpha$-syn aggregation, oxidative stress has often been reported to lead to the formation of insoluble cytotoxic $\alpha$-syn species (Vijayakumaran et al., 2015). Therefore, it is crucial to understand the regulatory mechanisms of $\alpha$-syn related to its solubility and/or its aggregation post-translationally for elucidating PD neuropathology. $\alpha$-Syn is known to undergo numerous post-translational modifications (PTMs). For example, its phosphorylation, ubiquitination, nitration, acetylation, and SUMOylation have been reported to play numerous roles in modulating $\alpha$-syn aggregation and toxicity (Giasson et al., 2000; Shimura et al., 2001; Fujiwara et al., 2002; Kim et al., 2011b; Popova et al., 2015; Kleinknecht et al., 2016). Ubiquitinated $\alpha$-syn is typically targeted to proteasomal or lysosomal degradation (Pan et al.,

Received April 2, 2020; accepted July 21, 2020; First published September 4, 2020.

The authors declare no competing financial interests.

Author contributions: D.K.V., A.G., E.C., J.B.S., and Y.-H.K. designed research; D.K.V., A.G., L.R., E.C., E.J., D.W., and E.-G.J. performed research; Y.-H.K. contributed unpublished reagents/analytic tools; D.K.V., A.G., L.R., E.C., E.J., M.M., J.B.S., and Y.-H.K. analyzed data; D.K.V. and Y.-H.K. wrote the paper.

This work was supported by the National Institutes of Health Grant 5P20GM103653 (to Y.-H.K.).

Acknowledgements: We thank the kind donation of UBC9 transgenic mice from Dr. Yangja Lee-Wickner and Dr. John Hallenbeck at the National Institute of Neurological Disorders and Stroke for the study. We also thank Dr. Melissa Harrington (Director, Delaware Center for Neuroscience Research) for supporting this project, undergraduate students Gabriela Cabrera and Suhyun Nam for their efforts in data analysis in a blinded manner, and Carol Lazzara for proof-reading the revised manuscript.

Correspondence should be addressed to Dinesh Kumar Verma at dinesh. kr.v87@gmail.com or Yong-Hwan Kim at yhkim@desu.edu.

https://doi.org/10.1523/ENEURO.0134-20.2020 Copyright (C) 2020 Verma et al.

This is an open-access article distributed under the terms of the Creative Commons Attribution 4.0 International license, which permits unrestricted use, distribution and reproduction in any medium provided that the original work is properly attributed.
2008). Thus, impaired proteasome function enhances the aggregation of $\alpha$-syn, and ubiquitinated proteins including $\alpha$-syn have been shown to aggregate in SNpc neurons (Spillantini et al., 1997; Eriksen et al., 2003).

SUMOylation is a reversible covalent modification that conjugates a small ubiquitin-like modifier (SUMO) protein to lysine in SUMO consensus domain in three separate steps such as SUMO activating enzyme (SAE1), Ubc9 and SUMO-E3 ligase (Wilkinson and Henley, 2010). Ubc9 is an E2-SUMO conjugase that covalently attaches a SUMO protein to $\alpha$-syn at lysine residues if they are within a defined specific consensus site $(\psi-\mathrm{K}-\mathrm{x}-\mathrm{D} / \mathrm{E})$, where $\psi$ corresponds to a large hydrophobic residue, $\mathrm{K}$ stands for lysine, $x$ can be any amino acid, and D/E are glutamate or aspartate (Rodriguez et al., 2001; Sampson et al., 2001). SUMO tagging is known to enhance the solubility of conjugated proteins and may play a role in regulating the solubility of aggregation-prone proteins (Marblestone et al., 2006; Krumova et al., 2011). However, SUMOylation can be a major mechanism that counteracts ubiquitination by different E3 ubiquitin ligases (parkin) and regulates $\alpha$-syn degradation (Rott et al., 2017). SUMOylated proteins have also been detected in the halo of Lewy bodies, co-localizing with $\alpha$-syn in brains of patients with either PD or dementia with Lewy bodies (DLB; Kim et al., 2011b). Thus, SUMOylation and ubiquitination of $\alpha$-syn in Lewy bodies may be regulatory mechanisms, modulating its degradation reciprocally in PD pathologic aggregation. Interestingly, UBC9 overexpression was reported to increase the solubility of $\alpha$-syn and prevent methamphetamine (METH)-induced protein aggregation. Further, non-SUMO $\alpha$-syn mutants enhance their aggregation by impairing proteasomal and lysosomal degradation (Zhu et al., 2018). Since SUMOylation of target proteins has been posited as an important factor in the pathogenesis or progression of PD, we assessed the effect of SUMOylation on $\alpha$-syn for preventing its protein aggregation. The question of whether SUMOylation of $\alpha$-syn is a good or bad modulation remains unresolved (Eckermann, 2013). Hence, the causal relationship between SUMOylation and $\alpha$-syn aggregation/degradation and the relevance to $\alpha$-syn toxicity need to be investigated.

A previous study showed that Ubc9 overexpression increases the protein level of dopamine transporter (DAT) and further enhances the functional activity of DAT with decreasing ubiquitination and degradation of DAT (Cartier et al., 2019). Here, we demonstrate a comprehensive role for SUMOylated $\alpha$-syn in protein aggregation/degradation in vitro. Our results support that Ubc9-mediated SUMOylation is neuroprotective against MPTP (MPP+) or preformed fibrils (PFFs) of $\alpha$-syn-induced toxicities in vivo and in vitro (Volpicelli-Daley et al., 2011). Intriguingly, we found that SUMOylated $\alpha$-syn was refractory to normal lysosomal degradation, however, this aberrant degradation was not detrimental to $\alpha$-syn-mediated protein aggregation because of the increased solubility (Krumova et al., 2011). Our study strongly suggests that SUMOylation is a novel mechanism 
that plays a crucial role in regulating $\alpha$-syn solubility and degradation in dopaminergic cells, and it can be a therapeutic target for PD.

\section{Material and Methods}

\section{Animals}

Ubc9-overexpressing transgenic (Ubc9-Tg) C57BL/6 background mice $(30-45 \mathrm{~g})$ were received as a gift from John Hallenbeck at NINDS (Lee et al., 2011, 2014). All animal protocols were conducted in accordance with the United States Public Health Service Guide for the Care and Use of Laboratory Animals; all procedures were approved by the Institutional Animal Care and Use Committee (IACUC). Four or five animals per polyacrylic cage were housed with access to food and water ad libitum and were maintained in standard housing conditions, i.e., at room temperature (RT) $24 \pm 1^{\circ} \mathrm{C}$ and humidity 60 $65 \%$ with $12 / 12 \mathrm{~h}$ light/dark cycle.

\section{Animal groups and treatment}

All the Ubc9-Tg mice (males and females) are hemizygous and their wild-type (WT) siblings were used as controls. When mice were aged up to 11-12 months old, $25 \mu \mathrm{g} / \mathrm{g}$ body weight of MPTP (dissolved in $0.9 \%$ saline, Sigma) was injected once a day for seven consecutive days (Lazzara et al., 2015). Since MPTP toxicity is strain and age sensitive, the chronic injection to the same age mice was performed at the same time for minimizing the variation of MPTP toxicity ( $n=10$ per group). In the vehicle group, the same volume of $0.9 \%$ saline was injected $(n=10)$. Seven days after the last injection, mice were deeply anesthetized in an isoflurane chamber and intracardiac perfusion was done by using ice-cold $0.9 \%$ saline, followed by $150 \mathrm{~mm} \mathrm{NaCl} / 70 \%$ ethanol. After decapitation, whole brains were isolated. Half of each brain was isolated by brain-region, e.g., the striatum and brain stem, and stored at $-80^{\circ} \mathrm{C}$ for molecular/biochemical analyses including reactive oxygen species (ROS) measurement; the other half brain was postfixed in $4 \%$ paraformaldehyde (PFA) for immunohistochemical analysis.

\section{Cell lines with plasmids or RNAi treatment}

N27 rat dopaminergic parental cell line (SCC048, EMD Millipore) was used to generate enhanced green fluorescent protein (EGFP) or Ubc9-EGFP-overexpressing (Ubc9-OE) stable cell lines by transfection. Neomycin resistant plasmids containing EGFP (based on pEGFP vectors, Clontech; now Takara Bio USA) or Ubc9-EGFP (a gift from Jo Morris, King's College, London) were transfected to N27 cells using Lipofectamine 3000 (Thermo) as per the manufacturer's instructions. All cell lines, including EGFP, Ubc9-OE, and rat dopaminergic parental cell line (N27p), were cultured in RPMI 1640 (Invitrogen) cell culture media along with 10\% FBS (Atlanta Bio) and 1\% penicillin-streptomycin (Invitrogen) in a $5 \% \mathrm{CO}_{2}$ incubator at $37^{\circ} \mathrm{C}$. Forty-eight hours after transfection, G418 (Geneticin, Invitrogen) was added as a selection marker at the concentration of $500-1000 \mu \mathrm{g} / \mathrm{ml}$ for two weeks. Clusters of green fluorescent-positive (EGFP) cells were isolated and plated into $60 \mathrm{~mm}$ dishes. G418
$(500 \mu \mathrm{g} / \mathrm{ml})$ was added for another two weeks and then lowered to $200 \mu \mathrm{g} / \mathrm{ml}$ as previously reported (Cartier et al., 2019). To knockdown Ubc9 expression, N27p cells were transfected with dicer substrate siRNAs (RNAi-N013050.12.1 5'-UCCGUACAGUUACUAGUA-3' 3'-UCAAUGAUCAUCG GACCC-5', RNAi-N013050.12.3 5'-GUACGAUGAACGUG AUGA-3' 3'-CUVGGACUACUUGACCCU-5' or negative control NC1 purchased from IDT using Lipofectamine 3000 (Thermo), according to the manufacturer's directions. After the exposure overnight, NC1 or Ubc9-RNAitreated cells were exposed to MPP + or PFF for $24 \mathrm{~h}$, followed by cellular measurements including cell viability, cytotoxicity, ROS, and thioflavin T staining, etc.

\section{Cell culture and treatment}

MPP + (1-methyl-4-phenylpyridinium, Sigma) treatment was applied at concentrations of $160,320,640$, and 1280 $\mu \mathrm{M}$ for $24 \mathrm{~h}$. The PFFs of $\alpha$-syn was purchased from StressMarq Biosciences (SPR-324C) and its treatment was tested at $1,2,3,4$, and $5 \mu \mathrm{g} / \mathrm{ml}$ for $24 \mathrm{~h}$. Cells were also treated with cycloheximide (Chx; Sigma), protein synthesis inhibitor $(100,150$, and $200 \mu \mathrm{g} / \mathrm{ml})$ for $24 \mathrm{~h}$ and PMA, PKC-mediated lysosomal inducer $(2,4,6,8$, and 10 $\mu \mathrm{M})$ for $2 \mathrm{~h}$. Additional experiments were performed using $640 \mu \mathrm{M} \mathrm{MPP}+, 150 \mu \mathrm{g} / \mathrm{ml}$ of Chx, and $5 \mu \mathrm{M}$ PMA for $24-\mathrm{h}$ exposure. There were also co-treatments of chloroquine (lysosomal inhibitor; $10 \mu \mathrm{M}$ ) and MG132 (proteasomal inhibitor; $10 \mu \mathrm{M}$ ), also known as Z-Leu-Leu-Leu-al for $24 \mathrm{~h}$ (Cartier et al., 2019).

\section{Cell viability assay}

The conversion of MTT dye to soluble formazan is caused by mitochondrial dehydrogenase enzymes in live cells. The formazan is soluble in DMSO and produces purple/blue color that indicates the level of cell viability. The cell viability in the vehicle group of EGFP and Ubc9 cells was calculated as $100 \%$ of control. Cell viability was estimated by using the dye 3-(4,5-dimethylthiazol-2-yl)-2,5diphenyltetrazolium bromide (MTT), following the protocol described by Janezic et al. (2016). A total of 5000 cells were seeded in 96-well plates. After $1 \mathrm{~h}$, cells were treated with different concentrations of MPP + or PFF $(1 \mu \mathrm{g} / \mathrm{ml})$ for $24 \mathrm{~h}$. MTT $(5 \mu \mathrm{M})$ dye was added into the culture media and incubated at $37^{\circ} \mathrm{C}$ for $2 \mathrm{~h}$. The culture medium was removed, and $200 \mu \mathrm{l}$ of DMSO was added to each well. The purple-colored formazan was dissolved in DMSO and color intensity was measured at $570 \mathrm{~nm}$ with a reference wavelength of $630 \mathrm{~nm}$ by using a spectrophotometer (SpectraMax M5 ${ }^{\mathrm{e}}$, Molecular devices). The measured optical density is directly correlated with cell viability.

\section{Cytotoxicity assay}

By measuring the level of lactate dehydrogenase (LDH; a soluble cytosolic enzyme), released into the culture medium on cell lysis, the level of cytotoxicity was assessed. The cytotoxicity assay was performed by using Pierce LDH cytotoxicity assay kit (88954, Thermo), as per the manufacturer's protocol. Briefly, after treatment with different concentrations of MPP+ or PFF for $24 \mathrm{~h}$, the cell 
culture media were collected for LDH assay, and cells were further used for cell viability (MTT) assay. The protein concentration was measured by using Pierce Rapid Gold BCA protein assay kit (A5225, Thermo Fisher Scientific) as per the manufacturer's protocol. For LDH assay, $50 \mu \mathrm{l}$ of cell culture media per well was transferred into a new 96-well plate. Then, $50 \mu$ of reaction mixture was added to each well, and a plate was incubated at RT for $30 \mathrm{~min}$. After incubation, $50 \mu$ of stop solution was added to each well. Absorbance changes were measured at 490 and $680 \mathrm{~nm}$ by a spectrophotometer (SpectraMax M5e, Molecular Devices). To determine LDH activity (\%), 680$\mathrm{nm}$ absorbance values were subtracted from the readings at 490-nm absorbance, and then the normalized values were calculated in the scale of vehicle-treated control.

\section{ROS level measurement}

The level of ROS was assessed by using CellROX deep red reagent (C10422, Thermo Fisher Scientific) as the manufacturer's protocol instructed. Briefly, after treatment of MPP + or PFF, CellROX (5 $\mu \mathrm{M})$ was added into the cell culture medium in duplicate and incubated at $37^{\circ} \mathrm{C}$ for $30 \mathrm{~min}$. The cells were then washed with PBS and images were randomly captured using the EVOS FL Cell Imaging Systems (Invitrogen). After four to five independent experiments (total replicates: $n=8-10$ ), the intensity of red color was analyzed by a blinded rater using ImageJ.

\section{Thioflavin $\mathrm{T}$ and $\alpha$-syn staining}

For double staining, up to $4.5 \times 104$ N27p cells were seeded per well on poly-D-lysine-coated glass coverslips in six-well plates. After transient transfection using Lipofectamine 3000 with Ubc9-RNAi or NC1 treatment overnight, cells were treated with wheat germ agglutinin/Nacetylglucosamine (WGAVGluNAc, Sigma) for PFF $(1 \mu \mathrm{g} / \mathrm{ml})$ penetration as described by Volpicelli-Daley et al. (2011). Briefly, cells were fixed with 4\% PFA/PBS solution for 20 min and washed three times in PBS. Blocking and permeabilization was done using $2 \%$ bovine serum albumin (BSA) prepared in PBS-T (0.25\% Triton X-100) at RT for 1 $\mathrm{h}$. Then, cells were incubated with anti- $\alpha$-syn primary antibodies (1:250, MABN1817, Sigma-Millipore) at RT for $2 \mathrm{~h}$ and washed in PBS three times. The cells were treated with Alexa Fluor 647-conjugated secondary antibodies (1:1000; Molecular probes, Thermo Scientific) at RT for $1 \mathrm{~h}$ and washed in PBS three times. For thioflavin T staining, we have followed the procedures from the recent publication (Polinski et al., 2018) with minor modifications. Cells were incubated with $20 \mu \mathrm{m}$ thioflavin T (Sigma-Aldrich) solution at RT for $30 \mathrm{~min}$ and washed in PBS three times. After staining, cell images were randomly captured using fluorescence microscopy (EVOS, Invitrogen) using proper optical filters. As a negative control, the secondary antibody step or the thioflavin T step was omitted. After capturing random cell images, the relative fluorescent intensity of thioflavin T (green) or $\alpha$-syn (red) was compared with control (WGA/NAcGlu, 100\%) for assessing the effect of PFF or Ubc9-RNAi. All the experiments were independently run at least four times in duplicate ( $n \geq 8$ per group) and three to five images per well were captured for thioflavin T or $\alpha$-syn. Thus, total images $(n=24-40)$ per group were analyzed by a blinded rater.

\section{Immunocytochemistry}

N27 cells were seeded on poly-D-lysine-coated glass coverslips per well in 6-well plates. Cells were washed in ice-cold PBS without $\mathrm{Ca}^{2+}$ or $\mathrm{Mg}^{2+}$ (137 mM NaCl, 2.7 $\mathrm{mm} \mathrm{KCl}, 4.3 \mathrm{~mm} \mathrm{Na}_{2} \mathrm{HPO}_{4}$, and $1.47 \mathrm{~mm} \mathrm{KH}_{2} \mathrm{PO}_{4}$ ) for $5 \mathrm{~min}$ three times, followed by fixation with $4 \%$ PFA at RT for $20 \mathrm{~min}$ and washed in PBS for $5 \mathrm{~min}$ three times. Fixed cells were permeabilized with $0.1 \%$ Triton $\mathrm{X}-100$ and $0.5 \%$ BSA for $3 \mathrm{~min}$, followed by blocking with $5 \%$ BSA at RT for $2 \mathrm{~h}$. Then, cells were incubated with mouse anti$\alpha$-syn primary antibodies (1:500, MABN1817, SigmaMillipore) in $1 \%$ BSA/PBS at $4^{\circ} \mathrm{C}$ overnight. On the following day, cells were washed in $0.1 \%$ PBS-T (Triton X-100) three times for $5 \mathrm{~min}$ each and incubated with secondary goat anti-mouse Alexa Fluor 594 (1:1000; Invitrogen, Thermo Fisher Scientific) at RT for $1 \mathrm{~h}$. Cells were washed in PBS for $3 \times 5 \mathrm{~min}$, followed by a brief washing in ultrapure water. Coverslips were mounted on glass slides using ProLong Diamond antifade mounting medium (P36961, Thermo). Images were obtained using Zeiss 780 Multiphoton Confocal microscopy at the imaging core in our institution. All images were acquired with a PlanApochromat $40 \times / 1.4$ NA Oil DIC objective (Zeiss). Images were processed using Zeiss ZEN software and sampled at optimal pixel density in the $X$ and $Y$ with a line average of 4 , and a zoom factor of 1.2. Z-stacks were set up with optimal z-sectioning for optimal overlap and processed into maximum intensity projections.

\section{Immunohistochemistry}

A series of total coronal sections throughout the striatum and substantia nigra were sectioned in 16- $\mu \mathrm{m}$ thickness and collected in total four sets for mounting onto positively charged slides (Midwest Sci). For rehydration, slides were immersed into xylene for 5 min twice, followed by washing in serially diluted ethanol $(100 \%$ for $5 \mathrm{~min}$ twice; $95 \%$ for $5 \mathrm{~min}$; $70 \%$ for $5 \mathrm{~min}$; $50 \%$ for $5 \mathrm{~min}$ ) and rinsed with deionized water. Slides were washed in PBS for 5 min twice. Antigen unmasking was done by using Tris-EDTA Buffer (10 mm Tris-base, $1 \mathrm{~mm}$ EDTA solution, and $0.05 \%$ Tween 20; pH 9.0) at RT for $15 \mathrm{~min}$ and washed in PBS for 5 min three times. After blocking in $2 \%$ BSA at RT for 30 min, slides were incubated with anti-tyrosine hydroxylase (TH) antibody (1:1000; AB152, EMDMillipore) at $4^{\circ} \mathrm{C}$ overnight and washed in PBS-T for $5 \mathrm{~min}$ three times. Then slides were incubated with Alexa Fluor 647-conjugated goat secondary anti-mouse antibody (1:1000; Invitrogen, Thermo Fisher Scientific) at RT for $1 \mathrm{~h}$ and washed in PBS-T for $3 \times 5 \mathrm{~min}$. As a negative control, the primary antibody step was omitted. Coverslips were mounted on slides using ProLong Diamond antifade mounting medium (Thermo). Images were acquired using LSM 510 confocal microscope with Z-stacks $(0.5 \mu \mathrm{m})$ which were set up with optimal z-sectioning for optimal overlap at the internal imaging core. All images were 
acquired with a Plan-Apochromat $40 \times / 1$.4 NA oil DIC objective. Images were processed into maximum intensity projections using Zeiss ZEN software. Every fourth section was analyzed for measuring the intensity of TH staining in the striatum and for counting $\mathrm{TH}+$ neurons in the substantia nigra compacta (SNc) in a blinded manner.

\section{$\alpha$-Syn quantitative mRNA measurement}

Using quantitative real-time PCR (qRT-PCR; LightCyler 480Il, Roche), the level of $\alpha$-syn mRNA was determined with $\beta$-actin as a housekeeping gene. An equal number of N27 cells overexpressing either Ubc9 or EGFP was collected for RNA extraction using the TRIzol Reagent (Life Technologies). The experimental procedures were repeated, according to the recent report (Cartier et al., 2019) except for the primer information below: the parameters for the reactions were: $95^{\circ} \mathrm{C}$ for $10 \mathrm{~min}$, followed by 50 cycles of $95^{\circ} \mathrm{C}$ for $30 \mathrm{~s}, 60^{\circ} \mathrm{C}$ for $10 \mathrm{~s}$, and $72^{\circ} \mathrm{C}$ for 10 $\mathrm{s}$. The fluorescence was recorded during the $72^{\circ} \mathrm{C}$ step to determine the Crossing point $(\mathrm{Cp})$ value. The primers were chosen based on Primer3Plus software and synthesized by the Integrated DNA Technologies (IDT). The primers are: $\alpha$-syn forward 5'-GCAGTGAGGCTTATGAAA TGC-3'; $\alpha$-synuclein reverse 5'-AGGCTTCAGGCTCAT AGTCTTG-3'; $\beta$-actin forward 5'-AGCCATGTACGTAGC CATCC-3'; $\beta$-actin reverse $5^{\prime}$-CTCTCAGCTGTGGTGG TGAA- $3^{\prime}$. Each trial was performed in triplicate, and three trials were collected for total of nine independent samples for each gene.

\section{Chx protein chase}

EGFP or Ubc9 cells were equally plated $(1 \times 105$ cells/ plate) on 60-mm dishes and incubated with different concentrations of Chx $(100,150$, and $200 \mu \mathrm{g} / \mathrm{ml})$ in the culture media overnight. Additional experiments were performed using $150 \mu \mathrm{g} / \mathrm{ml} \mathrm{Chx}$ at different time points $(T=0,6,12$, 18 , or $24 \mathrm{~h}$ ). Cells were lysed using 250- $\mu$ I RIPA buffer [50 mм Tris, $100 \mathrm{~nm} \mathrm{NaCl}, 1 \% \mathrm{NP}-40,1 \mathrm{~mm}$ sodium fluoride (NaF), 2 mм sodium orthovandate $\left(\mathrm{Na}_{3} \mathrm{VO}_{4}\right), 10 \mathrm{~mm} \mathrm{NEM}$, $1 \mathrm{~mm}$ PMSF, and $1 \%$ protease phosphatase inhibitors] and sonicated briefly on ice for solubilization ( $3 \mathrm{~s}$ on and $10 \mathrm{~s}$ off for five cycles). Treatment or vehicle was added to the media at $\mathrm{T}=0 \mathrm{~h}$ and remained for the rest of chase studies, which was terminated as described previously (Cartier et al., 2019). Cell lysate was centrifuged at 15,000 $\times g$ for $10 \mathrm{~min}$, and supernatant was collected and separated by $4-20 \%$ SDS-PAGE gels (GenScript). $\alpha$-Syn was detected by immunoblotting as described below in Immunoblot analyses.

\section{Immunoprecipitation (IP)}

After treatment, cells were washed in $1 \times$ PBS twice and lysed in $250 \mu$ l of RIPA buffer with incubation for 15$20 \mathrm{~min}$. After brief sonication, cell extracts were centrifuged at $15,000 \times g$ for $10 \mathrm{~min}$. The supernatant was transferred into a fresh vial and protein concentration was measured by using Pierce Rapid Gold BCA Protein Assay kit (A53225, Thermo Fisher Scientific). An equal amount of protein $(500 \mu \mathrm{g})$ was taken for IP assay, and samples were precleared with $A / G$ beads (Santa Cruz Biotech) at $4^{\circ} \mathrm{C}$ for $1 \mathrm{~h}$. To remove $A / G$ beads, brief centrifugation was followed by collecting the supernatant for transferring to a fresh vial. Then, equal amounts of anti- $\alpha$-syn antibody (MABN1817, EMD-Millipore) were added to each sample and incubated at $4^{\circ} \mathrm{C}$ overnight. On the following day, equal amounts of $A / G$ beads were added to each sample. After $2 \mathrm{~h}$ of incubation, samples were collected and mixed with non-reducing sample loading buffer (39001, Thermo Fisher Scientific). All Immunoprecipitated samples were separated by $4-20 \%$ SDS-PAGE gels (GenScript) along with $5 \%$ total lysate as input. SUMO1, ubiquitin and $\alpha$-syn proteins were detected by immunoblotting as described below in Immunoblot analyses.

\section{Immunoblot analyses}

Protein samples were loaded on precast polyacrylamide gel (SurePAGE Bis-Tris, 4-20\%, 12 wells; GenScript) and transferred to PVDF membrane (Immobilon-P, EMD-Millipore) using Bio-Rad transfer apparatus. Membranes were incubated with anti- $\alpha$-syn antibody (1:2000; MABN1817, EMDMillipore), anti- $\alpha$-syn antibody for IP samples (1:1000; 610787, BD Biosciences), anti-SUMO1 (1:1000, sc-5308, Santa Cruz Biotech), or anti-ubiquitin (1:1000, sc-8017, Santa Cruz Biotech) at $4^{\circ} \mathrm{C}$ overnight. Equal loading was determined by stripping and re-probing against glyceraldehyde-3-phosphate dehydrogenase (GAPDH; 1:10,000; AM4300, Thermo Fisher Scientific). After washing, membranes were incubated with secondary anti-mouse IgG antibodies conjugated with $\operatorname{HRP}(1: 10,000)$ at RT for $2 \mathrm{~h}$. The PageRuler-prestained protein ladder was used to estimate protein molecular weights on immunoblots (Thermo 26616). Signals were developed in Immobilon Forte Western HRP substrate (EMD-Millipore) and detected under ChemiDoc iBright CL1000 (Invitrogen). The integrated density (intensity/area) of each band was measured and normalized by GAPDH (or $\alpha$-syn in IPs) and/or total protein loading labeled by Reversible protein stain kit for PVDF membranes (Thermo, 24585) as a loading control. Immunoblot images were converted into eight-bit grayscale images and avoided over-saturation. Equal areas corresponding to selected lanes were analyzed on each blot image using ImageJ software $(\mathrm{NIH})$. Splicing was implemented only for clarity purposes and the adjustment was performed using the original larger image.

\section{Sample preparation and data analysis for mass spectrometry}

Cell extracts from Ubc9-OE or EGFP N27 cells were IP'ed by $\alpha$-syn antibodies (MABN1817, EMD Millipore) as described above and then run in 10\% SDS-PAGE gel (GenScript). Three pieces per well were dissected based on the size of bands and each piece was separately rinsed once in $200 \mu \mathrm{l}$ of $\mathrm{ddH}_{2} \mathrm{O}$, twice in $200 \mu \mathrm{l}$ of $25 \mathrm{mM}$ ammonium bicarbonate in $50 \%(\mathrm{v} / \mathrm{v})$ acetonitrile, followed by $100 \mu \mathrm{l}$ of acetonitrile to dehydrate the isolated gel pieces, which were then lyophilized. The dry gel pieces were rehydrated in $150 \mu \mathrm{l}$ of $25 \mathrm{mM}$ ammonium bicarbonate containing $25 \mathrm{ng} / \mu \mathrm{l}$ trypsin (pH 8.0). After rehydration, 

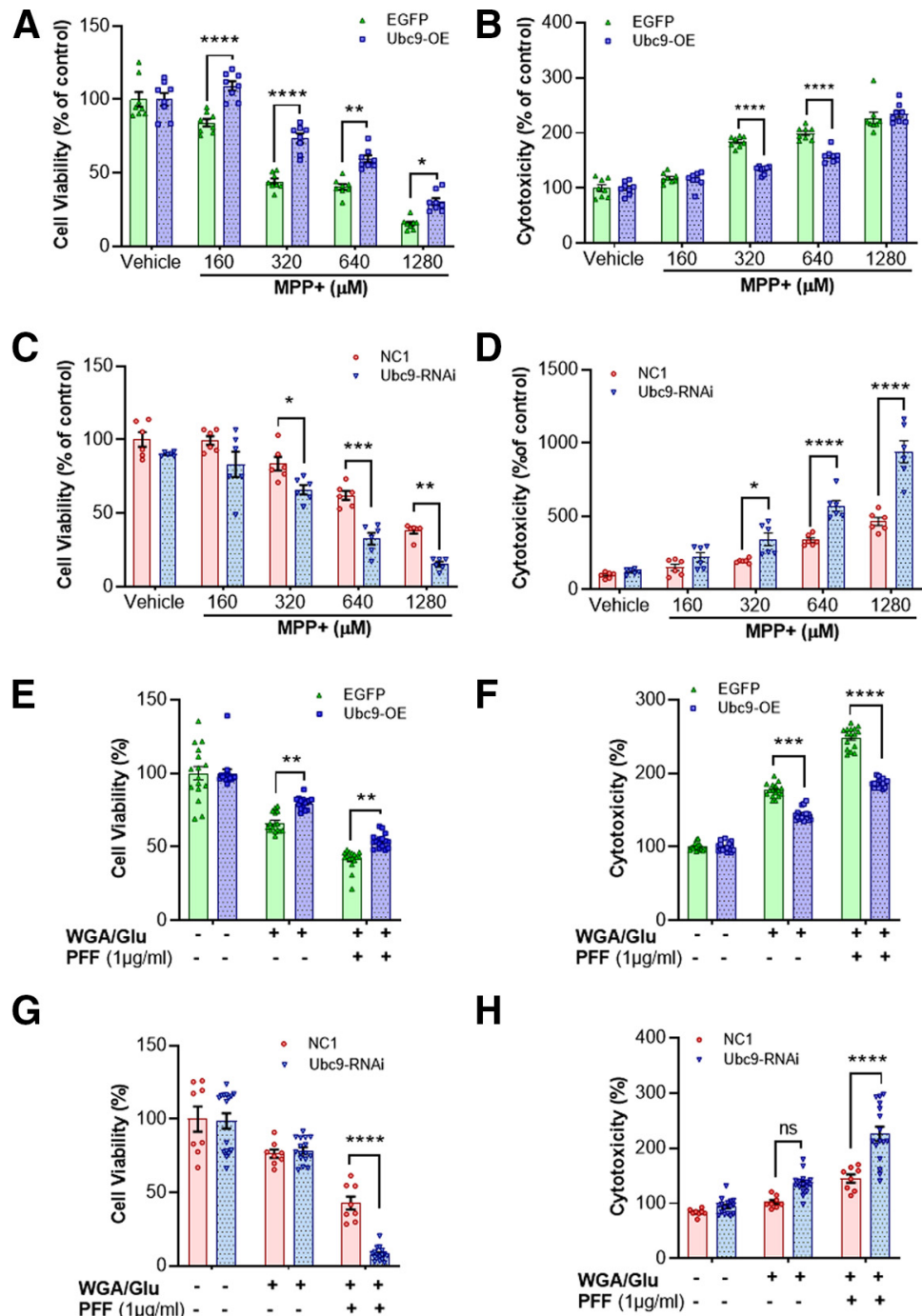

Figure 1. Ubc9 expression protects $\mathrm{N} 27$ cells from MPP + or PFF-induced toxicity, enhancing cell viability and reducing cytotoxicity. $\boldsymbol{A}, \mathrm{MPP}+$ exposure reduces the number of viable cells in EGFP cells in a dose-dependent manner, whereas Ubc9 overexpression protects N27 cells from MPP+ toxicity in MTT assay. B, In LDH assay, Ubc9 overexpression reduces the toxic effect derived from MPP+ exposure. $\boldsymbol{C}$, In MTT assay, Ubc9 knock-down by RNAi exacerbates the cell viability induced by MPP+ exposure, compared with NC1 random cocktail control. $\boldsymbol{D}$, In LDH assay, Ubc9-RNAi significantly increases the cytotoxicity derived from MPP+ treatment. $\boldsymbol{E}$, In MTT assay, PFF treatment reduces cell viability in EGFP cells compared with WGA/GluNAc-treated control, while Ubc9 overexpression ameliorates the toxic effect from PFF. $\boldsymbol{F}$, In LDH assay, PFF-induced cytotoxicity was suppressed by Ubc9 overexpression. G, In MTT assay, Ubc9-RNAi further exacerbates the cell viability induced by PFF treatment, compared with NC1 random cocktail control with WGA/GluNAc. $\boldsymbol{H}$, In LDH assay, Ubc9 knock-down substantially enhances the cytotoxicity derived from PFF treatment compared with NC1 control. All the treatments were exposed for $24 \mathrm{~h}$, and each dot represents the number of experiments and each experiment was performed in triplicate. Statistical analysis was applied using two-way ANOVA, Tukey's post hoc test. Scattered dot plots represent mean \pm SEM; $* p<0.05, * * p<0.01$, ***p $<0.001$, **** $p<0.0001$ ( $n=6-15$ per group).

additional $100 \mu \mathrm{l}$ of $25 \mathrm{~mm}$ ammonium bicarbonate was added and the gel pieces were incubated at $37^{\circ} \mathrm{C}$ overnight. Digested samples were desalted using a Harvard apparatus micro-spin column containing $\mathrm{C} 18$ resin. The pooled extracts were reduced to dryness and reconstituted in $80 \%$ acetonitrile/ $0.1 \%$ formic acid for tims-TOF Pro mass spectrometry (Bruker), which was operated in PASEF mode using Compass Hystar 5.0.37.1. The experiments were repeated three times for quantification $(n=3)$.

Data files were uploaded to PEAKS X (Bioinformatics Solutions) for processing, de novo sequencing and database search. The sequences were searched based on the UniProt Rattus norvegicus database $(37,157$ entries) since N27 cells are rat originated. We searched with mass error tolerances of $20 \mathrm{ppm}$ and $0.05 \mathrm{Da}$ for parent and fragment, respectively. Trypsin enzyme specificity, acetylation ( $\mathrm{N}$-term), oxidation, and phosphorylation were adopted as variable modifications. Peptides were filtered at an $1 \%$ false discovery rate $(p<0.01)$ in the peptide spectrum match level, and protein filtering was disabled by setting proteins as $-\log 10[\mathrm{P}]$ score at two unique peptides with a requirement for significant peptides. 
Table 1: Statistical analyses in Figures 1-4

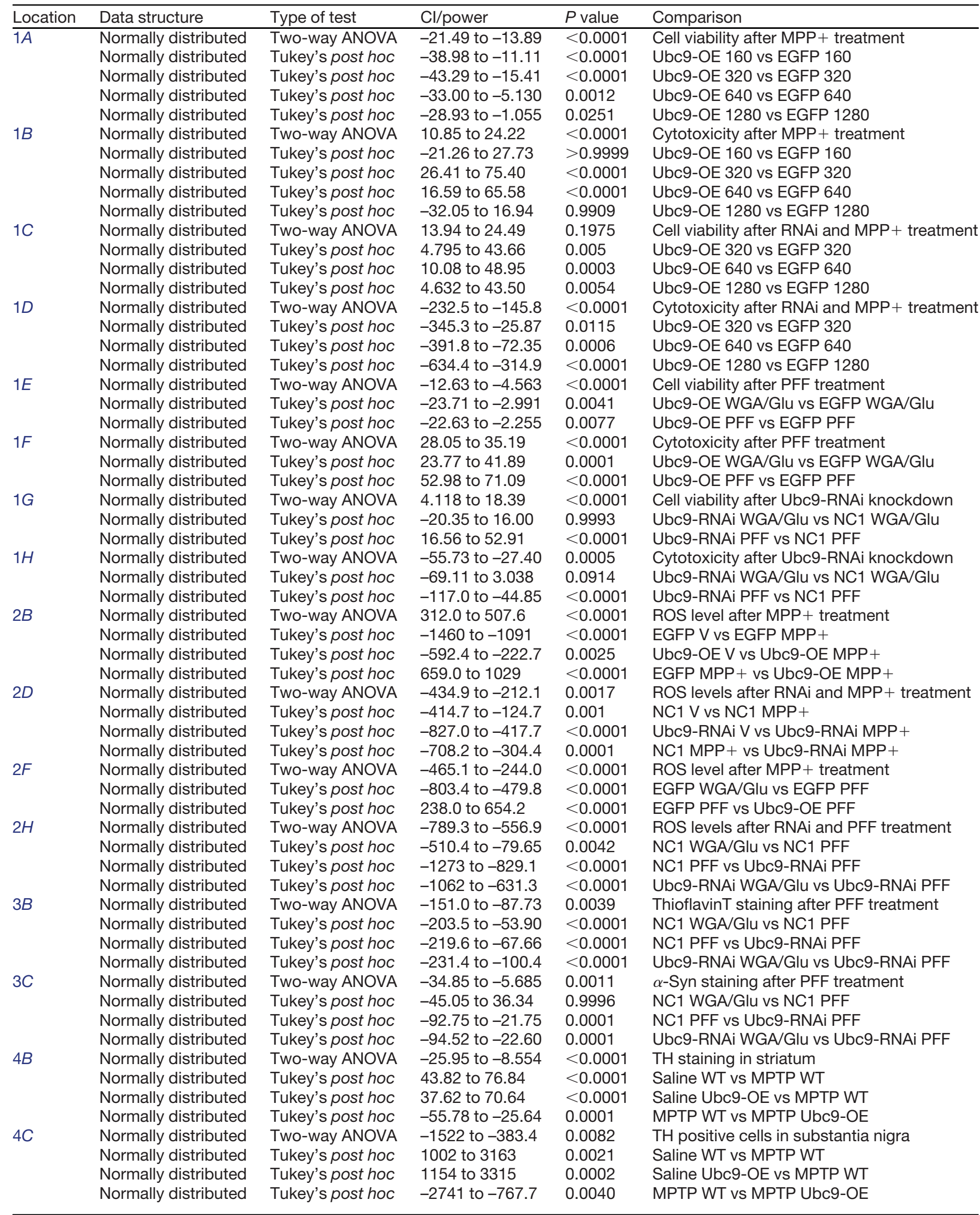


A

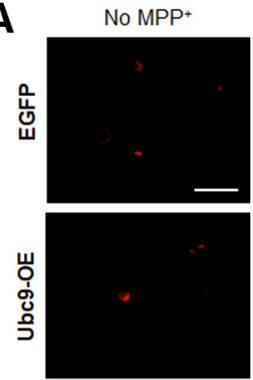

E

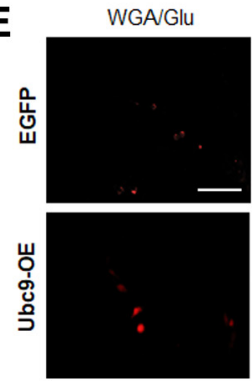

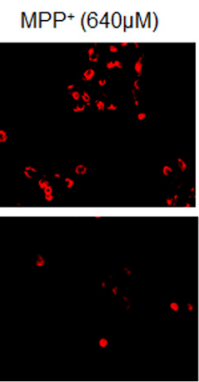
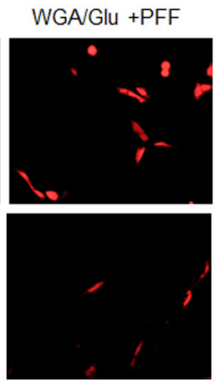

B

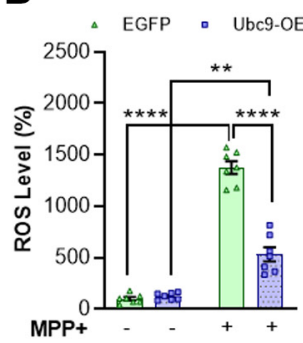

$F$

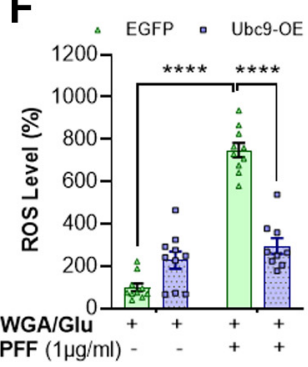

C

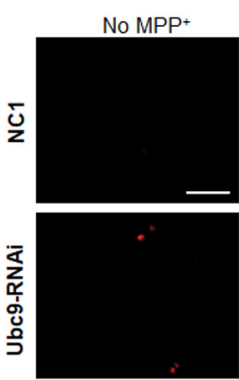

G

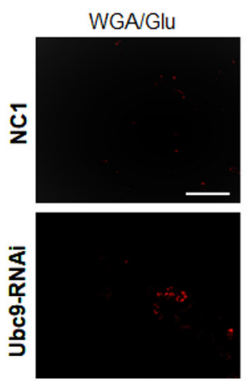

$\mathrm{MPP}^{+}(640 \mu \mathrm{M})$

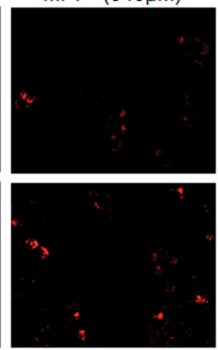

WGA/Glu +PFF

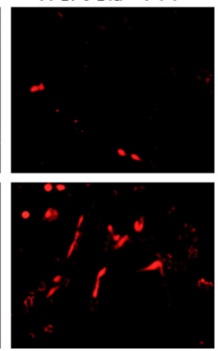

D
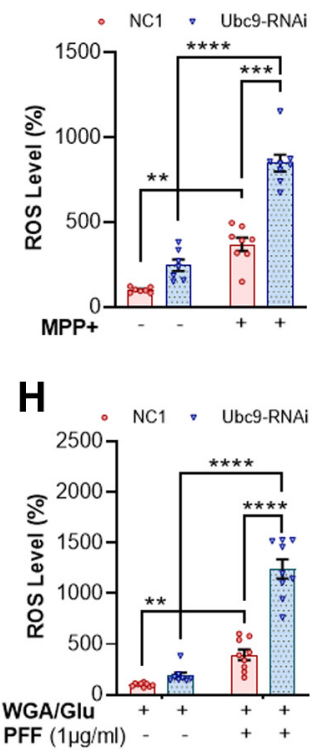

Figure 2. Ubc9 expression suppresses ROS generation triggered by MPP+ or PFF treatment. $\boldsymbol{A}$, Examples of ROS-labeled cells show that MPP + $(640 \mu \mathrm{M})$ for $24 \mathrm{~h}$ stimulates the generation of ROS in N27 EGFP cells, which was labeled with CellROX red fluorescent dye in dark field microscopy. $\boldsymbol{B}, \mathrm{MPP}+$ triggers a striking increase in ROS generation, which was prevented by Ubc9 overexpression. $\boldsymbol{C}$, Examples of cell images showing that Ubc9-RNAi exacerbates the ROS production induced by MPP + exposure. $\boldsymbol{D}$, The treatment of Ubc9-RNAi (100 pM) overnight enhances MPP+-induced ROS generation compared with NC1 random construct control. $\boldsymbol{E}$, Cell images show that PFF $(1 \mu \mathrm{g} / \mathrm{ml})$ exposure for $24 \mathrm{~h}$ stimulates ROS production (red label) in EGFP cells, which was not clearly detected with Ubc9 overexpression. $\boldsymbol{F}$, The ROS generation induced by PFF was almost completely suppressed by Ubc9 overexpression, compared with WGA/GluNAc control. G, Cell images show that Ubc9-RNAi exacerbates the PFF-induced ROS production, compared with no treatment or WGA/GluNAc control. $\boldsymbol{H}$, Ubc9-RNAi significantly enhances ROS production induced by PFF treatment, compared with NC1 control. Integrated density of images was measured by ImageJ and presented in scattered dot plots. Statistical analysis was applied using two-way ANOVA followed by Tukey's multiple comparisons post hoc test. All dot plots were displayed as individual values and mean differences are depicted as horizontal line $(n=7-10)$; SEM is indicated by the end of the vertical error bars; $* * p<0.01, * * * p<0.001, * * * * p<0.0001(n=8-10$ per group; $n$ represents the average intensity of ROS in each well). Scale bar: $20 \mu \mathrm{m}$.

\section{Chemicals}

Most chemicals in this study, such as phorbol 12-myristate 13-acetate (PMA; Sigma P1585), N-ethylmaleimide (NEM; Sigma E3876), Chx (Sigma C7698), chloroquine diphosphate (Sigma C6628), MG132 Z-Leu-Leu-Leu-al (Sigma C2211), lectin also known as WGA (Sigma L9640), $\mathrm{N}$-acetylglucosamine (Sigma PHR1432), MPP+ (Sigma D048), thioflavin T (T3516), MPTP (M0896), NaF (201154), and $\mathrm{Na}_{3} \mathrm{VO}_{4}$ (567540) were purchased from EMD-Sigma. PFF of $\alpha$-syn was obtained from StressMarq (SPR-324C) and PMSF (Roche 11359061001), BSA (Fisher bioreagent BP9703), and PBS (Invitrogen 10010031) were used as well. Protease inhibitor used was EDTA-free Halt protease inhibitor cocktail (Thermo Scientific 87785).

\section{Statistical analyses}

In most statistical analyses, two-way ANOVA was applied to assess the potential interaction between Ubc9-OE (or Ubc9-RNAi) and toxic treatment (MPP+ or PFF) in Figures 1-4, 9. In Figures 6-8, two-way ANOVA, Tukey's multiple comparison was also applied to analyze the interaction between Ubc9 and MG132 (or PMA), related to protein degradation. Student's unpaired $t$ test was applied to assess the Ubc9 effects, compared with EGFP only treatment (Fig. 5B,C). One- way ANOVA, Tukey's post hoc test was applied to Figure $5 E$, F. In Extended Data Figure 1-1, one-way ANOVA, Dunnett's test was adopted to compare different concentrations of treatment with vehicle control. For all studies, $p<$ 0.05 was considered statistically significant (*); GraphPad Prism 8.03 software was used for all data analyses and display. Values are presented as mean \pm SEM.

\section{Results}

\section{Cell viability and cytotoxicity}

MPP + treatment to both EGFP and Ubc9-EGFP cells decreased mitochondrial dehydrogenase activity with a gradient. After $24 \mathrm{~h}$ of MPP+ treatment at various concentrations (up to $1280 \mu \mathrm{M}$ ), cell viability in EGFP cells declined sharply in a dose-dependent manner, while that in Ubc9 cells decreased gradually compared with their vehicle groups. Ubc9 overexpression significantly ameliorated the toxic effects at all concentrations of MPP + compared with EGFP cells (Fig. 1A). Ubc9 cells showed significantly higher cell viability in terms of mean differences than EGFP cells at all the concentrations of MPP + (160-1280 $\mu \mathrm{M}$; Fig. 1A; Table 1). In LDH assays, MPP + treatment also increased cytotoxicity in both EGFP and Ubc9 cells as estimated by the release of cytosolic enzyme LDH in 
A

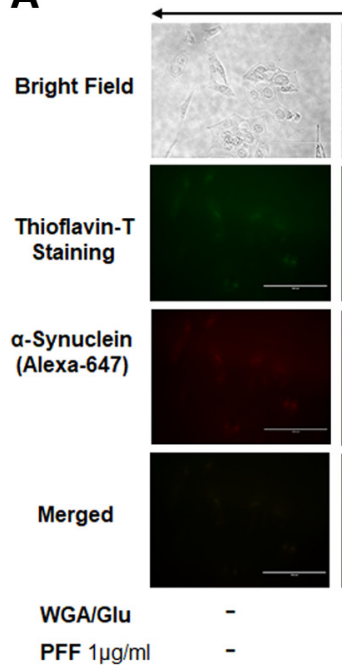

B

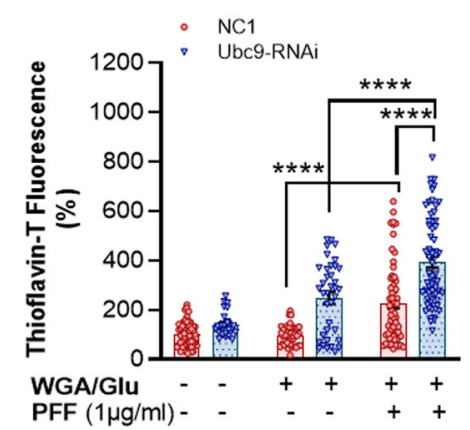

NC1
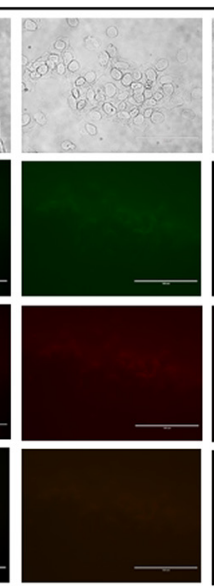

$+$
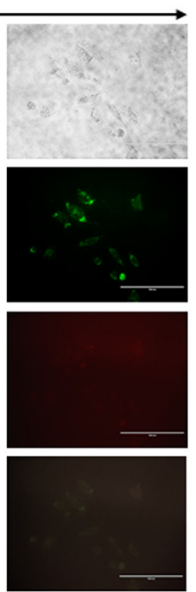

$+$

$+$
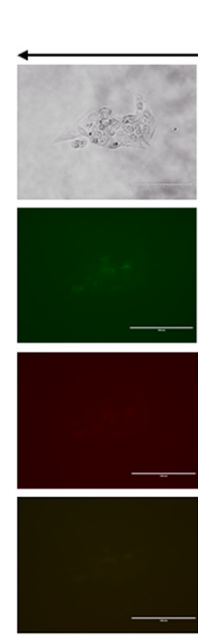

-
Ubc9-RNAi
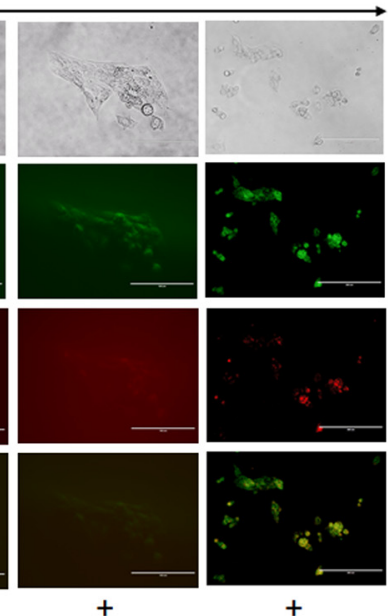

$+$

-

C

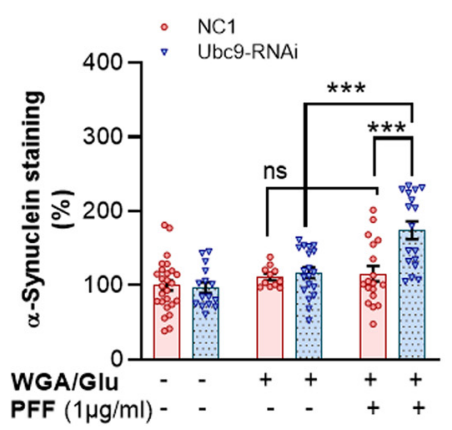

Figure 3. Ubc9 knock-down by RNAi exacerbates PFF-induced protein aggregation in thioflavin T staining. $\boldsymbol{A}$, Bright field images show the location of N27 cells (top row). Immunocytochemical images of thioflavin T staining in dark field show PFF-induced protein aggregation in green fluorescent label (second row). $\alpha$-Syn staining (red) in immunocytochemistry was detected in thioflavin T -labeled protein aggregates (third row). The merged images (yellow, the bottom row) demonstrate that $\alpha$-syn is co-localized with thioflavin T-stained protein aggregates. B, PFF exposure to N27 cells for 24-h results in the accumulation of protein aggregation labeled in thioflavin T. Ubc9-RNAi further enhances PFF-induced protein aggregation, compared with NC1 random cocktail control with WGA/GluNAc. $\boldsymbol{C}$, PFF increases the level of $\alpha$-syn in thioflavin T-positive aggregates with Ubc9-RNAi, and Ubc9 knock-down aggravates $\alpha$-syn accumulation in the protein aggregates compared with NC1 control after PFF treatment, although PFF did not significantly increase the level of $\alpha$-syn in the aggregates in NC1 control-treated cells. Integrated density of images was measured by ImageJ and presented in scattered dot plots. Statistical analysis was performed using two-way ANOVA followed by Tukey's multiple comparisons post hoc test. All dot plots were displayed as individual values and mean differences are depicted as horizontal lines; SEM is indicated by the end of the vertical error bars; $* * * p<0.001, * * * * p<0.0001$; ns: not significant ( $n=24-40$ per group). Scale bar: $100 \mu \mathrm{m}$.

culture medium. The LDH activity in the vehicle group of EGFP and Ubc 9 cells was calculated as $100 \%$ of control. After $24 \mathrm{~h}$ of MPP + treatment at various concentrations, cytotoxicity in EGFP cells was gradually elevated in a dose-dependent manner. In comparison with EGFP cells, Ubc9 cells showed $50.91 \%(p<0.0001)$ and $41.09 \%$ $(p<0.0001)$ less cytotoxicity in mean differences at the concentrations of 320 and $640 \mu \mathrm{M}$ MPP + , respectively. However, there were no significant differences at 160 and $1280 \mu \mathrm{M} \mathrm{MPP}+$, probably because of too little or too much LDH release by MPP+ at the concentration, respectively (Fig. 1B; Table 1).

Next, we applied various concentrations of MPP + as above (Fig. $1 A, B$ ) to Ubc9-RNAi-treated N27 cells for measuring cell viability and cytotoxicity. In MTT assays (Fig. 1C), we found that RNAi-mediated Ubc9 knock- down significantly exacerbated the MPP+-induced toxicity in N27 cells at 320,640 , and $1280 \mu \mathrm{M}$ MPP + (Table 1), compared with the random RNAi constructs control (NC1 from IDT). In LDH assays (Fig. 1D), we found the same patterns as cell viability assays that Ubc9-RNAi significantly enhanced the MPP+-mediated toxicity at 320 , 640 , and $1280 \mu \mathrm{M}$ MPP + (Table 1), compared with the NC1 control. Based on Figure $1 A-D, 640 \mu \mathrm{M} \mathrm{MPP}+$ was applied to additional experiments.

We also exposed N27 cells to the PFFs of $\alpha$-syn to assess the protective effect of Ubc9-mediated SUMOylation in MTT and LDH assays. First, we tested the PFF exposure to $\mathrm{N} 27$ cells in the range of $1-5 \mu \mathrm{g} / \mathrm{ml}$ to determine the optimal dose and demonstrate in the Extended Data Figure 11 that $1 \mu \mathrm{g} / \mathrm{ml}$ of PFF treatment was sufficient to induce significant toxicity in vitro as reported (Volpicelli-Daley et 

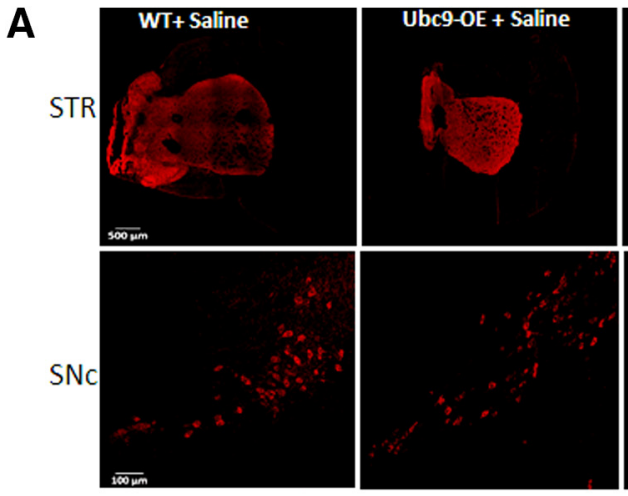

B

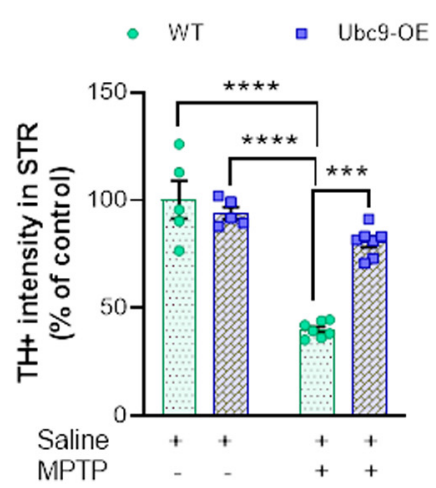

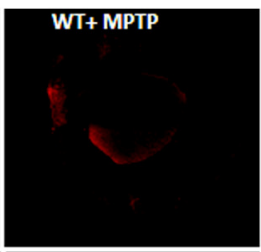
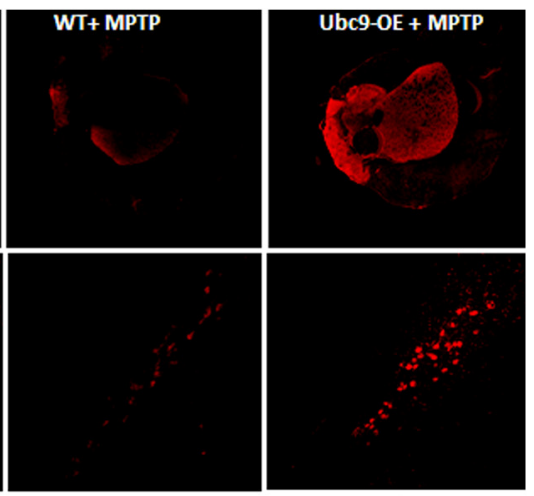

C

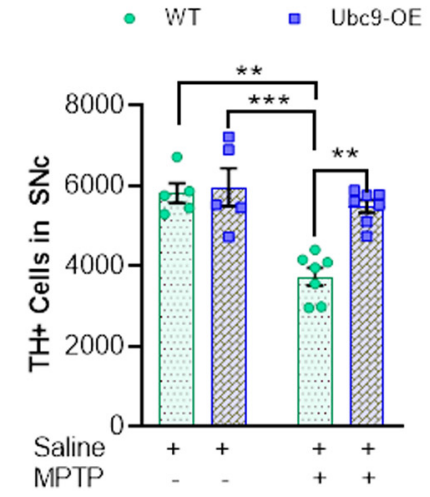

Figure 4. The overexpression of Ubc9 protects dopaminergic neurons from MPTP-induced toxicity in mouse brain. $\boldsymbol{A}$, Confocal images of the striatum (STR) and SNc region of C57BI/6 mice after staining with anti-TH antibody (1:100) conjugated to Alexa Fluor 555 (1:250). $\boldsymbol{B}$, Quantitative analysis of TH+ nigrostriatal projection intensity in the striatum of mouse brains. Dopaminergic projections in the STR of Ubc9-overexpressing Tg mice $(n=7)$ are significantly protected against MPTP toxicity, compared with WT-MPTP $(n=7)$. $\boldsymbol{C}$, The number of TH+ neurons in the SNc was significantly higher in Ubc9-Tg mice $(n=7)$ than that in WT siblings $(n=7)$, after chronic MPTP treatment. Dots represent values from individual mouse and horizontal bars represent mean \pm SEM. Statistical analysis was applied using two-way ANOVA, Tukey's test; $* * p<0.01, * * * p<0.001$, $* * * * p<0.0001$; size bars (500 $\mu \mathrm{m}$ for STR and $100 \mu \mathrm{m}$ for SNc).

al., 2011; Polinski et al., 2018). Next, using $1 \mu \mathrm{g} / \mathrm{ml}$ of PFF treatment, we tested the effects of Ubc9 overexpression or knock-down against PFF toxicity. Our results indicate that the overexpression of Ubc9 mitigates the PFF-induced toxicity compared with EGFP control cells. In Figure $1 E$, some level of cell damage was observed from WGA/ GluNAc only (PFF penetration mediator/blocker), but Ubc9 overexpression significantly protected N27 cells from PFFinduced toxicity in both cell viability $(p=0.0077$; Fig. $1 E)$ and cytotoxicity assays $(p<0.0001$; Fig. $1 F)$. In the following experiments, we applied PFF exposure to Ubc9-RNAitreated N27 cells and found that RNAi-mediated Ubc9 knock-down significantly enhanced the PFF-induced toxicity in N27 cells in both cell viability $(p<0.0001$; Fig. 1G) and cytotoxicity $(p<0.0001$; Fig. $1 H)$, compared with the random RNAi constructs control (NC1 from IDT).

\section{ROS levels}

MPP $+(640 \mu \mathrm{M})$ exposure for $24 \mathrm{~h}$ caused a drastic increase in ROS generation in both EGFP and Ubc9-EGFP cells. The base level of ROS increased 13.76-fold $(p<0.0001)$ in EGFP cells, whereas it increased 4.28 -fold in Ubc9 cells $(p=0.0025)$ by $640 \mu \mathrm{M}$ MPP + (Fig. $2 A, B)$. Thus, Ubc9 overexpression significantly suppressed MPP+-induced ROS generation when compared with
EGFP only $(p<0.0001$; Fig. $2 B)$. In the following experiments, we exposed Ubc9-RNAi to N27 parental cells overnight before MPP + treatment and then measured the level of ROS. We also found that Ubc9 knock-down enhanced MPP+-induced ROS generation almost 2.5-fold higher than NC1 control $(p<0.0001$; Fig. $2 C, D)$. In addition, we compared the level of ROS between Ubc9-overexpressing cells and EGFP cells after PFF $(1 \mu \mathrm{g} / \mathrm{ml})$ exposure for $24 \mathrm{~h}$ (Fig. 2E). PFF treatment increased $\sim 7$ fold higher ROS generation than the WGAVGluNAc control in EGFP cells $(p<0.0001)$, while it did not significantly increase ROS level in Ubc9-OE cells, compared with the WGAVGluNAc control. Thus, PFF-induced ROS generation was substantially suppressed by Ubc9 overexpression compared with EGFP controls $(p<0.0001$; Fig. $2 F)$. Furthermore, we measured the level of ROS with Ubc9RNAi and confirmed that Ubc9 knock-down robustly enhanced PFF-induced ROS generation compared with NC1 (PFF-treated group; $p<0.0001$; Fig. 2G,H). Although the PFF penetration accommodating reagent/blocker (WGA/GluNAc) increased ROS level subtly, compared with no treatment control, it was not statistically significant (data not shown). These results strongly support that Ubc9-mediated pan-SUMOylation reduces both MPP+-mediated and PFF-induced ROS production in vitro, explaining, at least in part, the protective effects by Ubc9-mediated SUMOylation shown in Figure 1. 

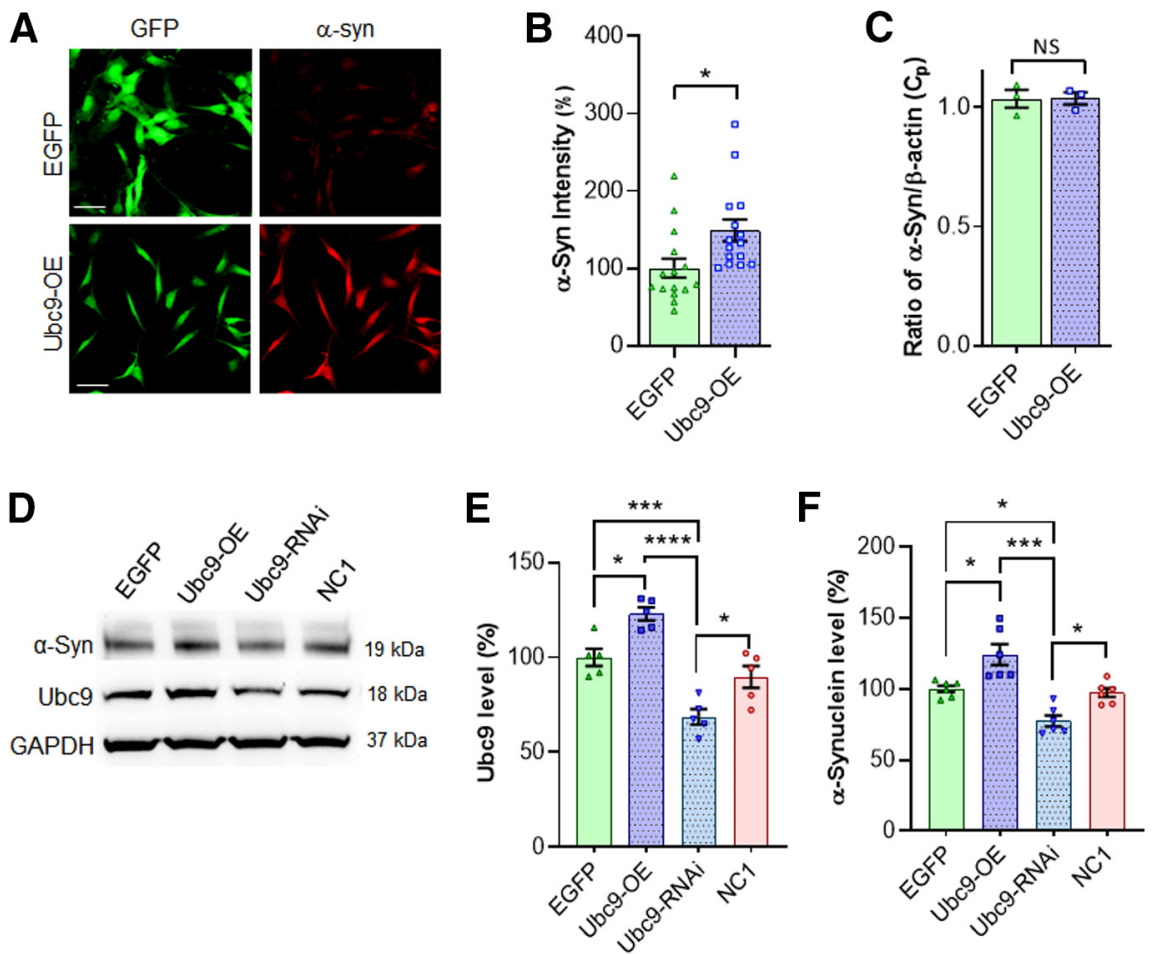

Figure 5. Ubc9 overexpression increases the endogenous level of $\alpha$-syn protein in dopaminergic N27 cells. $\boldsymbol{A}$, Immunofluorescent signals for $\alpha$-syn and EGFP in N27 cell lines stably expressing either Ubc9-EGFP or EGFP, are shown as indicated. Image scale bar: $20 \mu \mathrm{m}$. $\boldsymbol{B}$, In the quantification of fluorescence intensity of endogenous $\alpha$-syn in immunocytochemistry, higher level of $\alpha$-syn was detected in Ubc9 cells than in EGFP cells $(n=15)$. Statistical analysis was applied to Student's unpaired $t$ test. $\boldsymbol{C}$, In the qRTPCR, there is no difference in mRNA level of $\alpha$-syn between Ubc9 and EGFP cells $(n=3 \times 3)$. $\boldsymbol{D}$, In WBs, the levels of $\alpha$-syn and Ubc9 are displayed with the treatment of Ubc9-OE or -RNAi, in comparison to EGFP and NC1 controls. $\boldsymbol{E}$, Ubc9 expression was upregulated by Ubc9-OE and downregulated by Ubc9-RNAi in the quantification of WBs ( $n=5$ per group). $\boldsymbol{F}$, In the analysis of $24-\mathrm{h}$ chase assays, the mixed Ubc9-RNAi constructs significantly reduces the level of $\alpha$-syn, compared with the control (NC1) in WBs, whereas Ubc9-OE increases the level of $\alpha$-syn, compared with EGFP control $(n=6)$. Bars represent mean \pm SEM, and each dot represents the mean of each experiment, which was performed in triplicate. Statistical analysis was applied to one-way ANOVA, Tukey's post hoc test; $* p<0.05, * * * p<0.001$, $* * * * p<0.0001$; ns: not significant.

\section{Ubc9 knock-down by RNAi enhances PFF-induced protein aggregation in thioflavin T staining}

In order to assess the mechanisms of cellular protection by Ubc9 from PFF-induced toxicity, we also measured the level of protein aggregation, which is proportionally labeled in thioflavin T staining (Fig. $3 A$ ). As a PD model system, the PFF inoculation $(1 \mu \mathrm{g} / \mathrm{ml})$ triggers thioflavin Tlabeled protein aggregation in which $\alpha$-syn was detected (Fig. 3A, bottom row, merged). When N27 parental cells with NC1 were exposed to PFF treatment, a low level of protein aggregation was consistently detected in thioflavin $T$ staining (Fig. $3 A$, second row-third column). However, Ubc9-RNAi treatment aggravates the protein aggregation, which was clearly detected in thioflavin $\mathrm{T}$ staining (Fig. $3 A$, second row-sixth column). In quantitative analysis, PFF treatment resulted in protein aggregation in N27 parental cells with NC1, and Ubc9 knockdown by RNAi significantly enhanced the level of protein aggregation detected by thioflavin $T$ stain, compared with the NC1/WGA-GluNAc control treatment $(p<0.0001$; Fig. $3 B$ ). In addition, we also found that PFF treatment increased the level of $\alpha$-syn in protein aggregates with Ubc9-RNAi ( $p=0.0001$; Fig. $3 C$ ) and the treatment of Ubc9-RNAi increased the vulnerability of PFF-induced $\alpha$-syn accumulation in protein aggregates ( $p<0.001$; Fig. $3 C)$. However, the level of $\alpha$-syn in thioflavin T-stained protein aggregates in $\mathrm{NC1}$ was not significantly higher with PFF based on our analysis (Fig. 3C).

\section{Pan-Ubc9 overexpression protects dopaminergic neurons in the striatum and SNc from MPTP toxicity}

Coronal sections of mouse brain were stained by using anti-TH antibody (1:100) to detect dopaminergic neurons. MPTP exerts deleterious effects on dopaminergic neurons of WT C57BI/6 mice (Lazzara et al., 2015). Immunohistochemistry results showed a significant $(p<0.0001)$ decrease in $\mathrm{TH}+$ dopaminergic neuronal projections in the striatum region of MPTP-treated WT mice when compared with saline-treated WT or Ubc9 transgenic mice (Fig. 4A). After the confirmation of panUbc9 overexpression in brain from Ubc9-Tg mice in Western blottings (WBs; Lee et al., 2011), we found that the hemizygous Ubc9-Tg mice showed significant $(p=0.0001)$ protection in the striatum from MPTP toxicity, compared with a WT sibling group in the measurement of $\mathrm{TH}+$ density (Fig. 4A,B). Similarly, dopaminergic cell body count in the SNc was significantly reduced in 
A

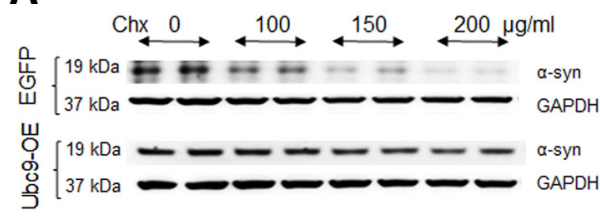

B

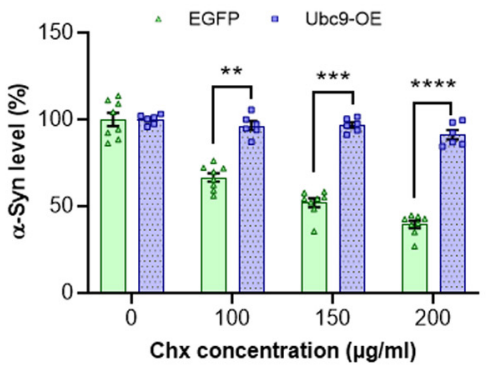

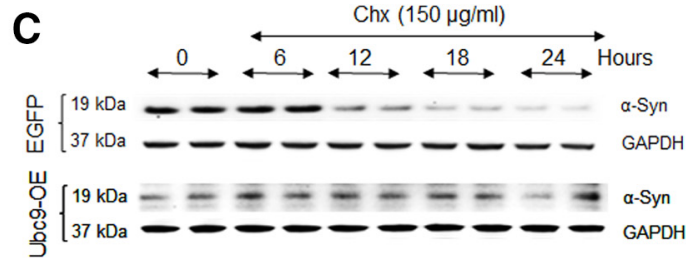

D

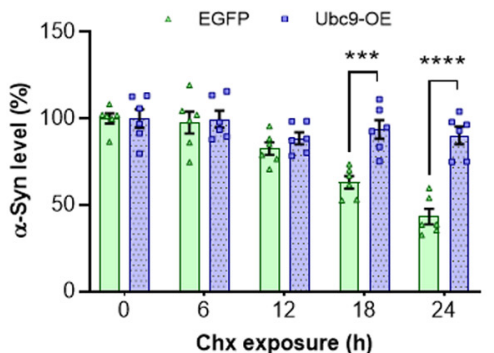

Figure 6. Ubc9 overexpression significantly prevents $\alpha$-syn degradation, compared with EGFP only in 24-h Chx-treated chase assays. $\boldsymbol{A}$, In WBs, the degradation of $\alpha$-syn was delayed by Ubc9 overexpression at different concentrations of Chx for $24 \mathrm{~h}$. $\boldsymbol{B}$, The protein level of $\alpha$-syn in N27-EGFP cells declines in the dose-dependent manner of Chx in 24-h chase study, whereas Ubc9-OE prevents the degradation of $\alpha$-syn. C, In WBs, the degradation of $\alpha$-syn in EGFP cells was detected in a time-dependent manner at various time exposures of Chx $(150 \mu \mathrm{g} / \mathrm{ml})$. $\boldsymbol{D}$, In the treatment of $150 \mu \mathrm{g} / \mathrm{ml}$ of Chx, >50\% of $\alpha$-syn was degraded in EGFP cells, while there is no significant decrease in Ubc9-OE cells at different time points up to $24 \mathrm{~h}$. Integrated density of WB images was measured using ImageJ. The ratio of $\alpha$-syn/GAPDH was presented as mean \pm SEM in $\%$ of control ( $n=6-8$ each time point). Statistical analysis was applied using two-way ANOVA, Tukey's post hoc test; $* * p<0.01, * * * p<$ $0.001, * * * * p<0.0001$.

MPTP-treated WT mice, compared with saline treated WT $(p=0.0021)$ or Ubc9 groups $(p=0.0002)$. However, Ubc9-Tg mice showed significant $(p=0.004)$ protection against MPTP-induced deleterious effects compared with MPTP-treated WT mice, in the analysis of two-way ANOVA, Tukey's test (Fig. 4A,C).

\section{Endogenous levels of $\alpha$-syn}

The SUMO conjugase Ubc9 is the main step to transfer SUMO1 and SUMO2/3 to target proteins. Therefore, we tested the effects of Ubc9 overexpression on endogenous levels of $\alpha$-syn. As shown in Figure 5A, Ubc9 overexpression in N27 cells increased the endogenous level of $\alpha$-syn almost $49 \%$ when compared with EGFP cells in immunocytochemistry $(p=0.0142 ;$ Fig. $5 B)$. The endogenous mRNA level of $\alpha$-syn remained unchanged even with Ubc9 overexpression (Fig. 5C). In WB analyses after overnight cultures, we demonstrate that the protein level of Ubc9 was $\sim 25 \%$ upregulated by Ubc9 overexpression compared with EGFP control $(p=0.012)$ and almost $40 \%$ downregulated by Ubc9-RNAi compared with random cocktail controls (NC1; $p=0.0229$; Fig. 5D,E). In the quantification of $\alpha$-syn WBs, Ubc9 overexpression increased almost $25 \%$ of $\alpha$-syn protein level compared with EGFP control $(p=0.025)$, while the combined Ubc9 knock-down constructs substantially reduced $\alpha$-syn protein compared with NC1 control ( $p=0.029$; Fig. $5 F)$. This result indicates that the enhanced level of $\alpha$-syn protein in Ubc9 cells is not derived from the increased mRNA level of $\alpha$-syn, suggesting that SUMOylated $\alpha$-syn avoids normal protein degradation as it was demonstrated in the DAT study (Cartier et al., 2019).

\section{Ubc9 overexpression reduces $\alpha$-syn degradation rate}

In Figure 5B, we demonstrate that Ubc9 overexpression enhanced the protein level of $\alpha$-syn, which was not derived from transcriptional up-regulation (Fig. $5 C)$. We then tested to see whether Ubc9-induced SUMOylation prevents endogenous $\alpha$-syn degradation in $24 \mathrm{~h}$ chase analysis. In order to quantify the level of the protein degradation, we performed $\alpha$-syn WBs using different concentrations of the protein synthesis inhibitor, Chx to compare the remaining amount of $\alpha$-syn in Ubc9 cells with that in EGFP cells. After blocking new protein synthesis, the remaining amount of $\alpha$-syn treated with vehicle in EGFP cells is considered $100 \pm 12.79 \%$ as control, and the amount declines as the concentrations of $\mathrm{Chx}$ increase. In contrast, the levels of $\alpha$-syn in Ubc9 cells were barely affected by the increase of Chx concentration (Fig. $6 A)$. The degradation rate of $\alpha$-syn was substantially reduced in Ubc9 cells compared with EGFP cells, resulting in significantly higher levels of $\alpha$-syn in Ubc9 cells than in EGFP cells at 100,150 , and $200 \mu \mathrm{g} / \mathrm{ml}$ of Chx (Fig. 6B; Table 2).

Based on Figure $6 B$, the $150 \mu \mathrm{g} / \mathrm{ml}$ of $\mathrm{Chx}$ was applied to determine the optimal time point from total cell lysates (0-24 h; Fig. 6C). In WB analyses (duplicate, $n=3$ independently), $\alpha$-syn levels in EGFP cells declined gradually from $T=0$ (100 $\pm 12.99 \%)$ to $T=24 \mathrm{~h}(41.58 \pm 2.62 \%)$. A significant degradation of $\alpha$-syn was observed from EGFP 
Table 2: Statistical analyses in Figures 5-9 and Extended Data Figure 1-1

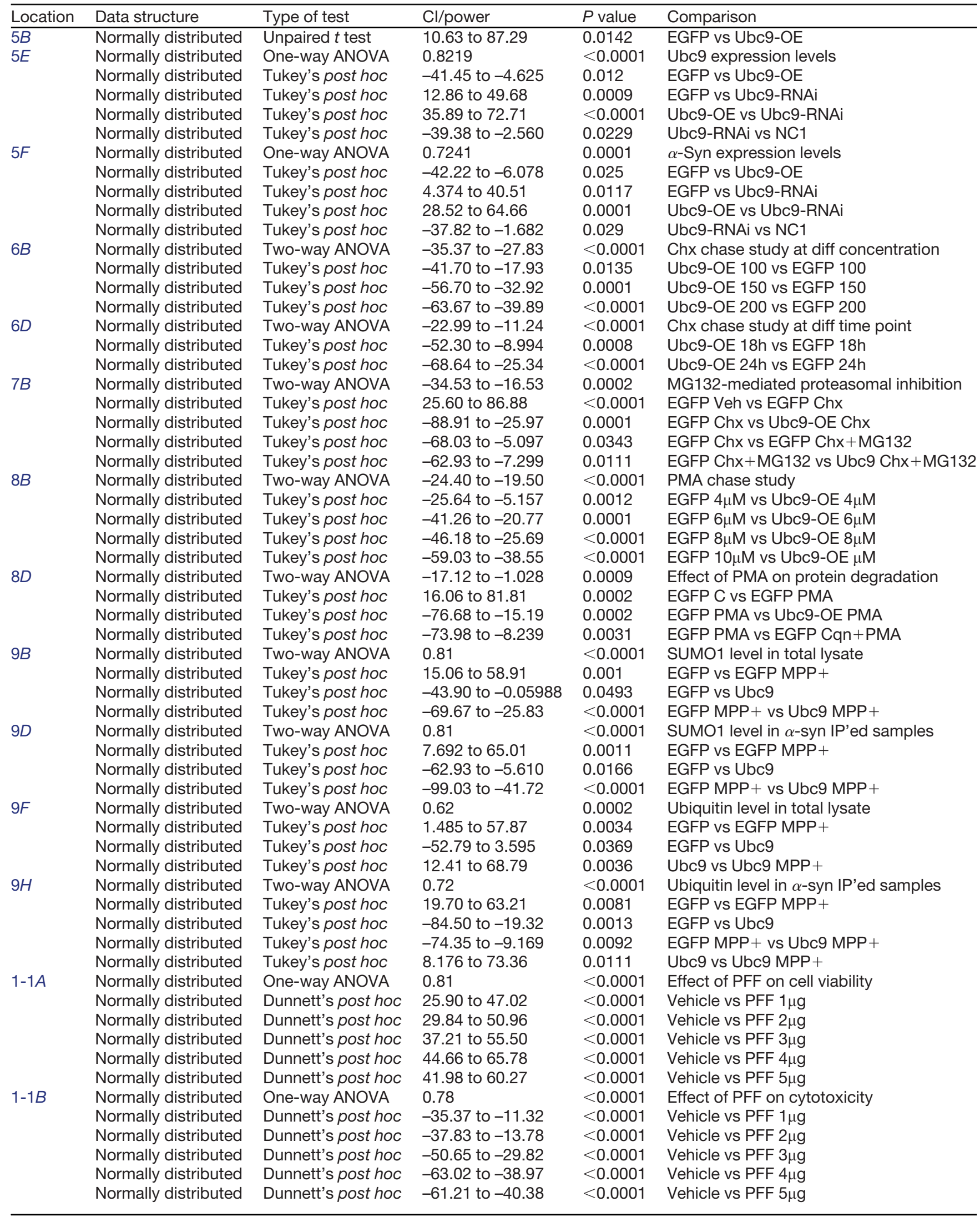



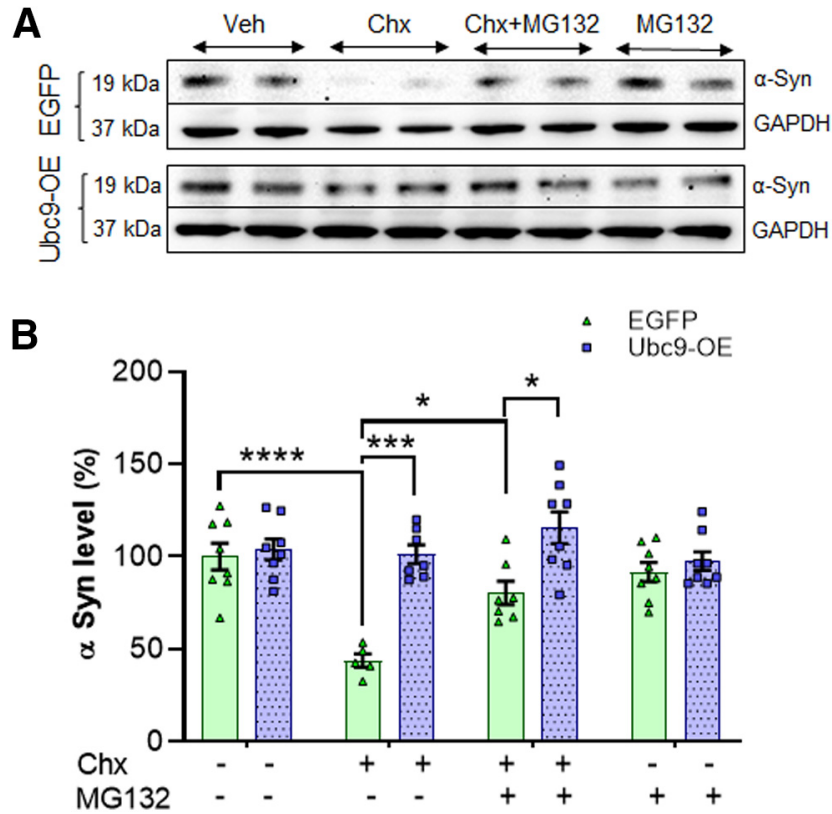

Figure 7. MG132-mediated proteasomal inhibition prevents $\alpha$-syn degradation in EGFP cells, while it shows no effect in Ubc9 cells in 24-h Chx chase studies. $\boldsymbol{A}$, In WBs, there is no noticeable effect of MG132 (proteasome inhibitor) in Ubc9 cells, compared with EGFP cells. $\boldsymbol{B}$, In the analysis of integrated density of WB images, $\alpha$-syn degradation was significantly interrupted by Ubc9 overexpression compared with EGFP cells. The ratio of $\alpha$-syn/GAPDH was displayed as mean \pm SEM in $\%$ of control ( $n=6-8$ each). Two-way ANOVA was applied to assess the interaction between Ubc9 and MG132 for statistical analysis; $* p<0.05, * * * p<0.001, * * * * p<0.0001$.

cells at T=18 h $(p=0.0033)$ and $T=24 \mathrm{~h}(p=0.0007)$ compared with the level at $\mathrm{T}=0$ (Fig. $6 D$ ). There was no significant difference observed in Ubc9 cells after Chx treatment over $24 \mathrm{~h}$. When compared with EGFP cells, the mean difference of the remaining $\alpha$-syn level in Ubc9 was $36.74 \%$ higher $(p=0.0008)$ at $T=18 \mathrm{~h}$ and $52.01 \%$ higher $(p<0.0001)$ at $\mathrm{T}=24 \mathrm{~h}$ (Fig. $6 D)$. Altogether our results indicate that Ubc9 overexpression reduces the degradation rate of $\alpha$-syn.

\section{Testing the mechanism of delayed $\alpha$-syn degradation by SUMOylation, through proteasomal pathway}

In the following experiment, we assessed the mechanisms of delayed $\alpha$-syn degradation, affected by Ubc9mediated SUMOylation. We tested to see whether the interruption of endogenous $\alpha$-syn degradation in Ubc9overexpressing cells results from the inhibition of the ubiquitin-proteasome system (UPS; Yan et al., 2005). After 24-h exposure of the proteasome inhibitor MG132 $(10 \mu \mathrm{m})$ in $\mathrm{Chx}(150 \mu \mathrm{g} / \mathrm{ml})$-treated chase studies, the endogenous level of $\alpha$-syn was measured in both Ubc9 and EGFP cell lines using WBs (Fig. 7A). Massive degradation of $\alpha$-syn was detected after Chx treatment $(p<0.0001)$; this was substantially reversed by MG132 co-treatment $(p=0.0343)$ in EGFP cells (the level of $\alpha$-syn at $24 \mathrm{~h}$ : vehicle $=$ $100 \pm 7.25 \%$; Chx $=46.07 \pm 10.32 \% ;$ Chx + MG132 $=$
$75.41 \pm 10.55 \%$; MG132=91.63 \pm 5.20\%; Fig. 7B) However, in Ubc9-OE cells, there was no significant change in the level of $\alpha$-syn after Chx and/or MG132 treatment (Fig. 7B, $n=4$ per treatment with duplicates). In our analysis, Ubc9 overexpression alone prevented $\alpha$-syn degradation by the same level as MG132 proteasomal inhibitor in EGFP control cells. Hence, there was no room to show any additive effect by MG132 or to detect the interaction between Ubc9 and MG132 in two-way ANOVA analysis. Therefore, we decided to test the effects of Ubc9 on $\alpha$-syn degradation via the Iysosomal pathway.

\section{Ubc9 overexpression blocks the lysosomal degradation of $\alpha$-syn induced by PMA treatment}

$\alpha$-Syn is known to be degraded by UPS and the autophagy-lysosome pathway (ALP); however, it is more likely degraded inside lysosomes through chaperonemediated autophagy (CMA) or endocytosis (Webb et al., 2003; Cuervo et al., 2004; Martinez-Vicente and Vila, 2013). Therefore, we assessed the capacity of Ubc9 overexpression to impair PMA-induced $\alpha$-syn degradation through lysosomal degradation (Cartier et al., 2019). We performed Chx chase analyses in the presence of PMA (PKC activator for inducing lysosomal degradation) with or without chloroquine, a known lysosomotropic inhibitor that reduces lysosomal protease activities (Lu et al., 2017). In quantitative WB analysis, we measured how much Ubc9 overexpression prevents the PKC-mediated lysosomal degradation of $\alpha$-syn. In Figure $8 A, B$, we assessed the optimal concentration of PMA in the range of 0-10 $\mu \mathrm{M}$ and confirmed that PMA induced lysosomal degradation of $\alpha$-syn in EGFP cells in a dose-dependent manner. However, there was no significant effect of PMA in Ubc9 cells (Fig. 8B). Based on the results in Figure 8B, we found that $5 \mu \mathrm{M}$ PMA for $2 \mathrm{~h}$ was sufficient to trigger lysosomal degradation of $\alpha$-syn in EGFP cells. The PKC activation by PMA $(5 \mu \mathrm{M})$ for $2 \mathrm{~h}$ induced a significant $\alpha$-syn degradation in EGFP cells $(p=0.0002)$, compared with vehicle only $(\alpha$-syn level: vehicle $=93.92 \pm 6.19 \%$; PMA $=$ $51.06 \pm 9.43 \%$; chloroquine $+\mathrm{PMA}=92.17 \pm 4.15 \%$; Fig . $8 D$ ). The lysosomal degradation of $\alpha$-syn induced by PMA was blocked by co-incubation with the lysosomal protease inhibitor, chloroquine $(p=0.0031)$. The residual level of $\alpha$-syn in Ubc9 cells was not significantly affected by PMA treatment because of the robust prevention of $\alpha$-syn degradation by Ubc9 overexpression (Fig. $8 C, D$ ). Therefore, the interaction between Ubc9 and PMA or the additive effect by PMA was not detected in two-way ANOVA, suggesting that Ubc9-mediated SUMOylated $\alpha$-syn almost completely avoids PMA-induced lysosomal degradation.

\section{Ubc9 overexpression prevents the MPP + -induced SUM01 removal from $\alpha$-syn}

In the following experiments, we assessed the regulatory mechanisms of $\alpha$-syn by SUMO1 or ubiquitin. In Figure $9 A$, we detected robust expressions of SUMO1 from total cell lysates and found that Ubc9 

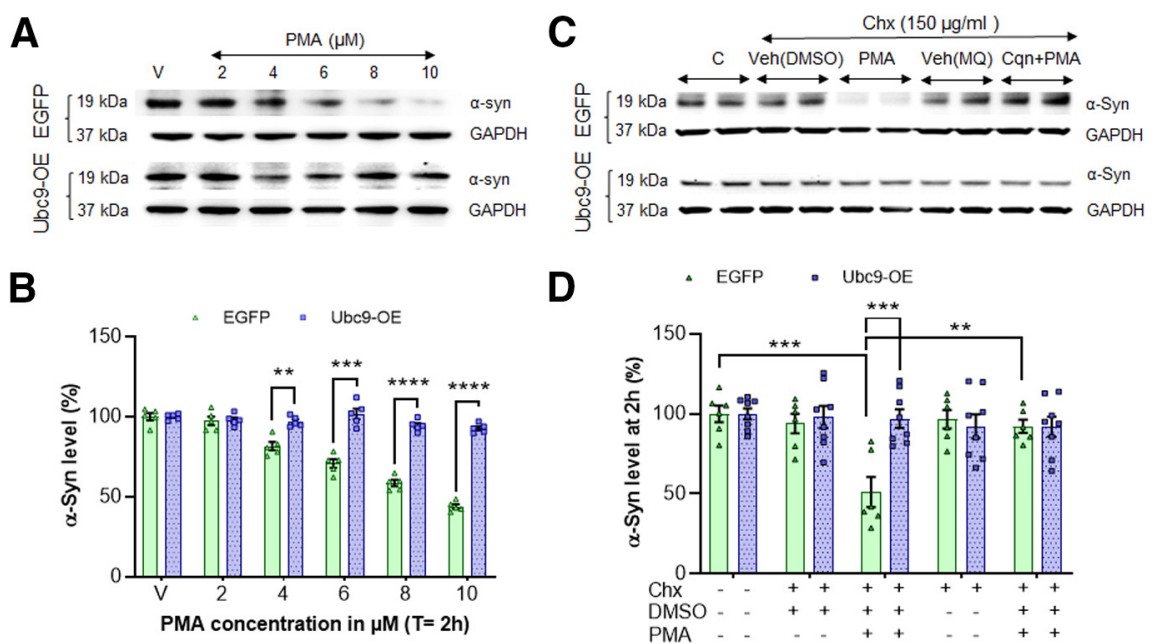

D

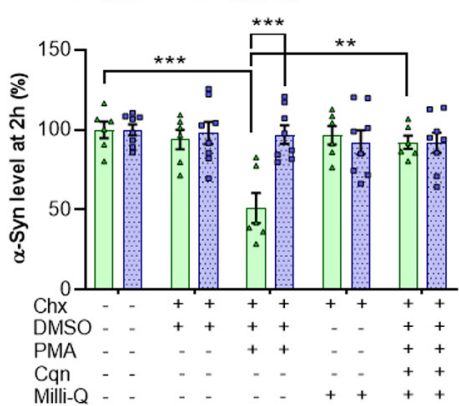

Figure 8. Ubc9 overexpression significantly prevents the PMA-induced lysosomal degradation of $\alpha$-syn in 2-h protein degradation analysis. $\boldsymbol{A}$, In WBs, the level of $\alpha$-syn in N27-EGFP cells declines in the dosage-dependent manner of PMA treatment in 2-h chase study, while that in Ubc9-OE cells remains the same. $\boldsymbol{B}$, In the integrated density analysis of WB images, Ubc9-OE significantly prevents PMA-induced degradation of $\alpha$-syn even at $10 \mu \mathrm{M}$ PMA $(n=5)$. $\boldsymbol{C}$, After the optimization of PMA and Chx at various concentrations, $5 \mu \mathrm{M}$ PMA and $150 \mu \mathrm{g} / \mathrm{ml}$ of Chx were applied to EGFP and Ubc9 cells for WBs. $\boldsymbol{D}$, In the integrated density analysis of WB images, PMA in EGFP cells substantially induces the degradation of $\alpha$-syn in 24-h chase study, while Ubc9 overexpression prevents the PMA-induced lysosomal degradation of $\alpha$-syn. No treatment control $(\boldsymbol{C})$ was considered as $100 \%$ in the relative ratio of $\alpha$-syn/ GAPDH and DMSO was vehicle for PMA treatment. The second vehicle for the PMA inhibitor, chloroquine (Cqn) was distilled water $\left(\mathrm{MQ}\right.$, Milli-Q $\left.\mathrm{H}_{2} \mathrm{O}\right)$, which was included as well as Cqn+PMA for comparison. The ratio of $\alpha$-syn/GAPDH was presented in mean \pm SEM in \% of control ( $n=5-8$ per treatment). Statistical analysis was performed by using two-way ANOVA to assess the interaction between Ubc9 and PMA; $* * p<0.01, * * * p<0.001, * * * * p<0.0001$

overexpression increased the total amount of SUMO1 expression compared with EGFP cells $(p=0.0493)$, as expected. When N27 cells were exposed to MPP+, the level of SUMO1 was significantly reduced in EGFP cells $(p=$ 0.001 ), but not decreased in Ubc9-overexpressing cells (ns; Fig. 9B). Thus, the level of SUMO1 was significantly higher in Ubc9 cells than that in EGFP cells $(p<0.0001$; Fig. 9B). As expected, we found streaked band patterns even with IP'ed $\alpha$-syn, probably because of various forms of SUMO1 binding to $\alpha$-syn (Fig. 9C). After $\alpha$-syn was IP'ed, we found the same pattern that SUMO1 was removed from $\alpha$-syn by MPP + in EGFP cells $(p=0.0011)$, in contrast, the level of SUMO1 on $\alpha$-syn in Ubc9-OE cells was not affected by MPP+ (Fig. 9D). Similar to Figure 9B, the level of SUMO1 on $\alpha$-syn in Ubc9 cells was significantly higher than that in EGFP cells $(p<0.0001$; Fig. 9D).

According to the mass spectrometry-based relative quantification of protein interaction with IP'ed $\alpha$-syn, the level of ubiquitin on $\alpha$-syn was 2.2-fold higher in Ubc9 cells than in EGFP cells (Extended Data Figure 9-1). Thereafter, we measured the levels of ubiquitin in total cell lysates as well as $\alpha$-syn IP'ed samples using WBs. Although the level of ubiquitin was higher from Ubc9 cell lysates than that from EGFP cell lysates without MPP+ exposure, MPP + significantly reduced the total level of ubiquitin in EGFP cells $(p=0.0034)$ as well as in Ubc9 cells (Fig. 9E,F; Table 2). Similarly, the level of ubiquitin in $\alpha$-syn IP'ed samples was noticeably reduced by MPP + exposure in both EGFP and Ubc9 cells. However, the base level of ubiquitin-bound $\alpha$-syn was substantially higher in Ubc9 cells than in EGFP cells, and the pattern remained the same with MPP + treatment (Fig. 9G,H; Table 2). Taken together, these results suggest that Ubc9 overexpression enhances not only the level of SUMO1 on $\alpha$-syn, but it also increases the level of ubiquitin on $\alpha$-syn. However, Ubc9 overexpression prevents the MPP+-induced SUMO1 removal from $\alpha$-syn, while it does not affect the ubiquitin removal from $\alpha$-syn.

\section{Discussion}

In this study, we demonstrate that Ubc9 overexpression protected dopaminergic cells from MPP+- or PFF-induced toxicity in vitro and further, pan-Ubc9 overexpression prevented dopaminergic neuronal loss in the striatum and SNc from MPTP-induced toxicities. The mechanism of SUMO-mediated neuroprotection may be, at least in part, derived from the prevention of ROS generation induced by MPP+ or PFF (Fig. 2). In addition, PFF treatment enhanced protein aggregation labeled in thioflavin $T$ and Ubc9-RNAi exacerbated PFF-induced protein aggregates containing $\alpha$-syn (Fig. 3B). Although high levels of $\alpha$-syn in NC1 control were consistently detected in the thioflavin T-positive protein aggregates after PFF exposure, this trend was not shown to be statistically significant (Fig. 3C). Ubc9 overexpression enhanced the level of SUMOylation on $\alpha$-syn, and SUMOylated $\alpha$-syn was refractive to residual protein degradation without affecting transcriptional up-regulation, whereas Ubc9-RNAi reduced the protein level of $\alpha$-syn (Fig. 5). Taken together, our results strongly support the report by Krumova et al. (2011) that SUMOylated $\alpha$-syn promotes its solubility, 
A

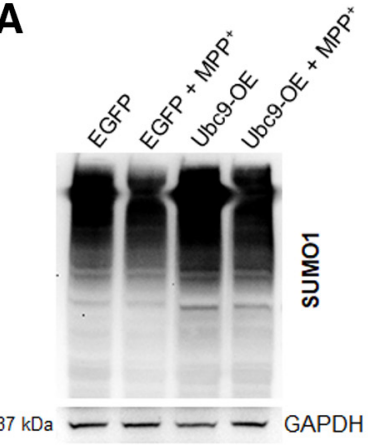

B

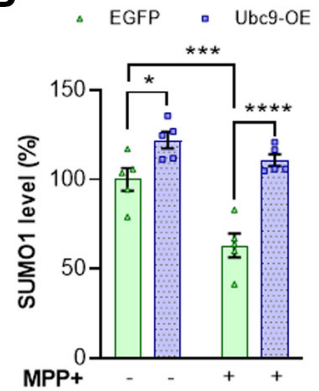

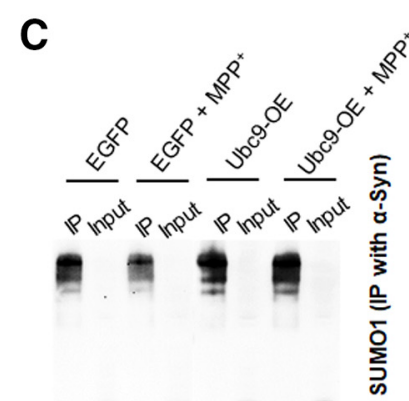

$19 \mathrm{kDa}-\quad-\quad-\alpha-S y n$

D

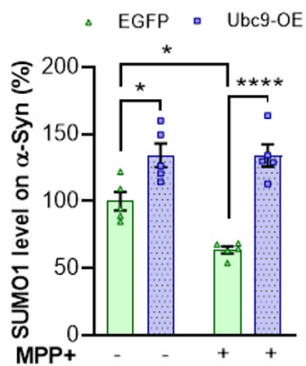

E

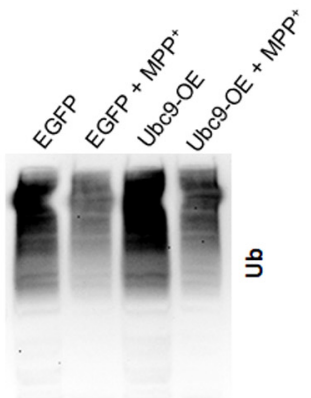

$37 \mathrm{kDa}-\sim-\mathrm{GAPDH}$

$\mathbf{F}$

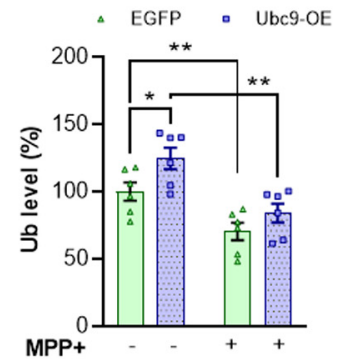

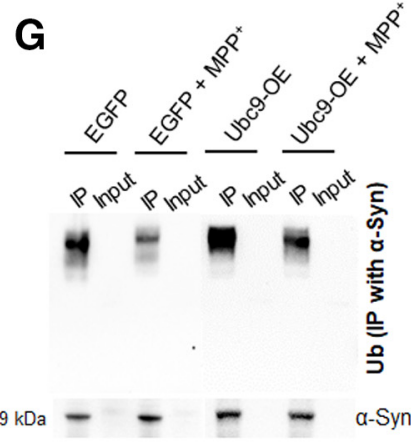

H

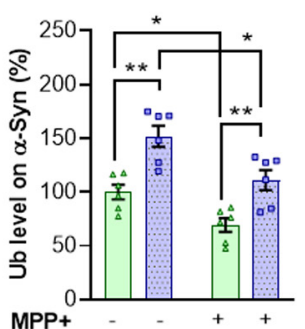

Figure 9. Ubc9 overexpression enhances the levels of SUMO1 and ubiquitin on $\alpha$-syn, while it prevents MPP+-induced SUMO-1 removal from $\alpha$-syn. $\boldsymbol{A}$, WB images show that the level of SUMO1 was increased with Ubc9 overexpression from total cell lysates. GAPDH was used as a loading control for total cell lysates. $\boldsymbol{B}$, MPP + exposure significantly reduces the total amount of SUMO1 in N27-EGFP cells, while Ubc9-OE prevents MPP+-induced SUMO1 down-regulation in total cell lysates. C, In WB using IP'ed $\alpha$-syn, MPP + reduces the level of SUMO1 bound to $\alpha$-syn in EGFP cells. $\alpha$-Syn was used as a loading control after IPs. $\boldsymbol{D}$, Ubc9 overexpression prevents MPP+-induced SUMO1 removal from $\alpha$-syn in quantitative WB analysis. $\boldsymbol{E}$, Examples of WB show that the total amount of ubiquitin $(\mathrm{Ub})$ was reduced by MPP + treatment. GAPDH was used as a loading control for total cell lysates. $\boldsymbol{F}$, The levels of ubiquitin from total cell lysates were decreased by MPP+ exposure both in EGFP and Ubc9 cells. $\boldsymbol{G}$, In WB, the levels of ubiquitin bound to $\alpha$-syn were substantially reduced by MPP + treatment. $\alpha$-Syn was used as a loading control after IPs. $\boldsymbol{H}$, MPP + exposure results in a significant reduction of ubiquitin bound to $\alpha$-syn in both EGFP and Ubc9 cells. MPP $+(640 \mu \mathrm{M})$ treatment for $24 \mathrm{~h}$ was applied to all experiments. Integrated density of WB images was measured using ImageJ. The ratios of SUMO1 or ubiquitin over respected loading control were presented as mean \pm SEM in \% of control. Statistical analysis was applied using two-way ANOVA, Tukey's multiple comparison post hoc test; $* p<0.05$, ** $p<0.01, * * * p<0.001$, ***** $<0.0001$ ( $n=5-8$ each group).

prevents protein aggregation and further, reduces cytotoxicity in the SNpc. Our findings also support the recent publication that METH exposure reduces the level of SUMOylation on $\alpha$-syn, and METH-induced $\alpha$-syn aggregation is relieved by Ubc9 overexpression (Zhu et al., 2018). The study has also demonstrated that mutations in SUMOylation acceptor sites in $\alpha$-syn enhance $\alpha$-syn overexpression and aggregation induced by METH, which is mediated by impaired degradation through the UPS and the ALP in vitro and in vivo (Zhu et al., 2018). As our results suggest that diminished levels of SUMOylation are more prone to aggravate PFF-induced protein aggregation, it is in line with the recent DAT study (Cartier et al., 2019).

As shown in Figure 1, cell viability of Ubc9-overexpressing cells was significantly higher and its cytotoxicity was significantly lower than those of EGFP only cells after various concentrations of MPP+ or PFF exposure for $24 \mathrm{~h}$. Although the mechanisms of suppressing ROS generation by pan-SUMOylation need to be characterized, there are numerous reports showing that SUMOylation plays a role in reducing oxidative stress. For example, SUMOylation may be an important regulatory mechanism that indirectly represses the production of ROS to ameliorate cellular stress (Pandey et al., 2011) and protects against oxidative stress with attenuation of stress-induced ROS generation by NADPH oxidase 2 (NOX2) inhibition (Kim et al., 2011a). Similarly, both conditional ablation and overexpression of Ubc9 induce functional impairment in mouse pancreatic $\beta$ cells via interrupting ROS detoxification derived from NRF2 activity (He et al., 2018). Furthermore, the chemical inhibition of Ubc9 by 2-D08 induces ROS accumulation, which stimulates apoptosis in acute myeloid leukemia cells, probably via NOX2 deSUMOylation (Zhou et al., 2019). In addition, SUMO1 plays a critical role in regulating proper mitochondrial dynamics by protecting DRP1 protein which is required for mitochondrial fission (Harder et al., 2004). Hayashi et al. (2002) showed that the apoptotic process was triggered when SUMO1 conjugation was impaired. Therefore, there is mounting evidence to support that SUMOylation plays a critical role in regulating detoxification from oxidative stress to prevent cell death.

Previous in vivo studies have shown that animals with higher SUMO conjugation levels are more resistant to ischemic insult (Lee et al., 2011, 2014). In their study, Lee et 
al., modeled several lines of transgenic mice whose Ubc9 expression was broadly elevated to various degrees (Lee et al., 2011). These transgenic mice were observably more resistant to permanent middle cerebral artery occlusion (pMCAO), an animal stroke model, than corresponding WT animals. Higher Ubc9 levels in the brain resulted in lower infarction volumes under pMCAO (Lee et al., 2011). An in vitro study demonstrated that SUMOylation of a small portion of $\alpha$-syn is sufficient to suppress its aggregation (Abeywardana and Pratt, 2015), which supports the previous study that a deficiency in the SUMOylation of $\alpha$-syn augments its aggregation and thereby increases deleterious cellular toxicity (Krumova et al., 2011). However, other studies revealed the opposite conclusion that SUMOylation facilitates $\alpha$-syn aggregation by blocking its ubiquitin-dependent degradation pathway and promoting its accumulation (Rott et al., 2017). Additionally, SUMO labeling of Lewy bodies in tissues from patients with PD and DLB was reported by Kim et al. (2011b), suggesting that SUMO was recruited to $\alpha$-syn inclusions induced by proteasome inhibition. To resolve the discrepancy, we tested the effects of SUMOylaton on dopaminergic neurons against MPTP using pan-Ubc9-overexpressing $\mathrm{Tg}$ mice (Lee et al., 2011). In Ubc9-Tg mice, the deleterious effects by oxidative stress were strikingly reduced, as indicated that $\mathrm{TH}+$ cell number in the $\mathrm{SN}$ and their projections to the striatum were significantly higher than those in WT mice, similarly treated with MPTP. As a follow-up, we also measured the levels of ROS from brain tissues to confirm our own in vitro results (Fig. 2); however, we could not detect meaningful ROS levels because of the lack of freshness of brain tissues. Because of quick redox reactions, we had technical difficulties in collecting reasonable levels of ROS from brain samples.

Our study demonstrates that Ubc9 overexpression increased the levels of SUMO1 and ubiquitin on $\alpha$-syn (Fig. 9) and prevented MPP+ (MPTP)-induced SUMO1 removal from $\alpha$-syn (in vivo data not shown); however, Ubc9 overexpression was not sufficient to block ubiquitin removal from $\alpha$-syn (Fig. 9). In the previous DAT study, we have suggested that SUMO1 and ubiquitin may compete with each other to bind to lysine residues on DAT, although this hypothesis may be applied to certain proteins specifically (Cartier et al., 2019). More relevant to PD pathology, SUMOylation and ubiquitination may regulate $\alpha$-syn degradation and pathologic aggregation reciprocally, suggesting its detrimental regulation by the competition (Rott et al., 2017). However, in our study, Ubc9 overexpression-induced SUMOylation may not counteract the ubiquitin binding to $\alpha$-syn since the ubiquitin level of $\alpha$-syn was upregulated by Ubc9 overexpression, not downregulated by the potential competition. Thus, the competition between SUMO and ubiquitin for binding to lysine residues in $\alpha$-syn may be varied to regulate proteostasis, depending on the experimental conditions. In addition, the potential cross talk between deSUMO enzymes, sentrin-specific proteases (SENPs), and deubiquitinating enzymes (Ub-proteases) can be explored to understand the possible indirect competition (Liebelt and Vertegaal,
2016). Furthermore, the interplay between SUMOylation and phosphorylation in $\alpha$-syn can be another interesting competition for preventing protein aggregation, although their targets are different amino acids (Shahpasandzadeh et al., 2014). Intriguingly, our results still support that SUMOylation delays the degradation of $\alpha$-syn, yet it is not a pathologic process but rather prevents pathologic aggregation because of its high solubility (Krumova et al., 2011; Gupta et al., 2014).

In the previous study, we found that Ubc9 overexpression is independent of proteasomal degradation, yet it effectively prevents the PMA-mediated lysosomal degradation of DAT. The enhanced levels of DAT in the plasma membrane contribute to high functional activity of DAT in dopamine uptake (Cartier et al., 2019). Moreover, in our current $\alpha$-syn study using the Chx-treated chase analysis, we verified that Ubc9 overexpression almost completely prevented the degradation of $\alpha$-syn. The aberrant degradation was more likely mediated by the suppression of PKC-mediated lysosomal degradation than by the inhibition of proteasomal degradation, since the effect of PMA was annulled by Ubc9 overexpression, while the lack of MG132 effect was detected (Figs. 7,8; Sun et al., 2014). However, the inhibition of potential proteasomal degradation by SUMOylation cannot be excluded because MG132 may not be able to contribute to the additional inhibition because of the saturated level of Ubc9 effect. Therefore, we do not exclude the possible interruption by Ubc9 overexpression for proteasomal degradation of $\alpha$-syn. Although Ubc9-mediated prevention of protein degradation may not occur exclusively through the lysosomal pathway and may be regulated by protein specificity, the inhibition of lysosomal degradation by SUMOylation was consistently identified in our DAT and $\alpha$-syn studies. As a follow-up to better understand the idiopathic mechanisms of PD pathology, we are currently characterizing how SUMOs are removed from $\alpha$-syn by PFF toxicity and determining which isoform of deSUMO enzymes, SENPs, is involved in detaching SUMOs from $\alpha$-syn in the scope of understanding the idiopathic mechanisms of PD pathology (Liebelt and Vertegaal, 2016). These studies may reveal SUMOs or SENPs as novel regulatory targets that increase the protein solubility and prevent the formation of Lewy bodies in PD pathology.

\section{References}

Abeywardana T, Pratt MR (2015) Extent of inhibition of $\alpha$-synuclein aggregation in vitro by SUMOylation is conjugation site- and SUMO isoform selective. Biochemistry 54:959-961.

Burré J, Sharma M, Tsetsenis T, Buchman V, Etherton MR, Südhof TC (2010) Alpha-synuclein promotes SNARE-complex assembly in vivo and in vitro. Science 329:1663-1667.

Cartier E, Garcia-Olivares J, Janezic E, Viana J, Moore M, Lin ML, Caplan JL, Torres G, Kim YH (2019) The SUMO-conjugase Ubc9 prevents the degradation of the dopamine transporter, enhancing its cell surface level and dopamine uptake. Front Cell Neurosci 8:35.

Chandra S, Gallardo G, Fernández-Chacón R, Schlüter OM, Südhof TC (2005) Alpha-synuclein cooperates with CSPalpha in preventing neurodegeneration. Cell 123:383-396.

Choi BK, Choi MG, Kim JY, Yang Y, Lai Y, Kweon DH, Lee NK, Shin YK (2013) Large $\alpha$-synuclein oligomers inhibit neuronal SNARE-mediated vesicle docking. Proc Natl Acad Sci USA 110:4087-4092. 
Cuervo AM, Stefanis L, Fredenburg R, Lansbury PT, Sulzer D (2004) Impaired degradation of mutant $\alpha$-synuclein by chaperone-mediated autophagy. Science 305:1292-1295.

Eckermann K (2013) SUMO and Parkinson's disease. Neuromolecular Med 15:737-759.

Eriksen JL, Dawson TM, Dickson DW, Petrucelli L (2003) Caught in the act: $\alpha$-synuclein is the culprit in Parkinson's disease. Neuron 40:453-456.

Fujiwara H, Hasegawa M, Dohmae N, Kawashima A, Masliah E, Goldberg MS, Shen J, Takio K, Iwatsubo T (2002) Alpha-synuclein is phosphorylated in synucleinopathy lesions. Nat Cell Biol 4:160164.

Giasson BI, Duda JE, Murray IV, Chen Q, Souza JM, Hurtig HI, Ischiropoulos H, Trojanowski JQ, Lee VM (2000) Oxidative damage linked to neurodegeneration by selective $\alpha$-synuclein nitration in synucleinopathy lesions. Science 290:985-989.

Gupta MK, Gulick J, Liu R, Wang X, Molkentin JD, Robbins J (2014) Sumo E2 enzyme UBC9 is required for efficient protein quality control in cardiomyocytes. Circ Res 115:721-729.

Harder Z, Zunino R, McBride H (2004) Sumo1 conjugates mitochondrial substrates and participates in mitochondrial fission. Curr Biol 14:340-345.

Hayashi T, Seki M, Maeda D, Wang W, Kawabe Y, Seki T, Saitoh H, Fukagawa T, Yagi H, Enomoto T (2002) Ubc9 is essential for viability of higher eukaryotic cells. Exp Cell Res 280:212-221.

He X, Lai Q, Chen C, Li N, Sun F, Huang W, Zhang S, Yu Q, Yang P, Xiong F, Chen Z, Gong Q, Ren B, Weng J, Eizirik DL, Zhou Z, Wang CY (2018) Both conditional ablation and overexpression of E2 SUMO-conjugating enzyme (UBC9) in mouse pancreatic beta cells result in impaired beta cell function. Diabetologia 61:881895.

Janezic EM, Caviness JE, Kanda SG, Davis XD, Kim YH (2016) Commercially available pesticides cause additive or synergistic damages in dopaminergic cells: relevance for Parkinson's disease pathology. Ann Neurodeg Dis 1:1010.

Jin H, Kanthasamy A, Ghosh A, Yang Y, Anantharam V, Kanthasamy AG (2011) $\alpha$-synuclein negatively regulates protein kinase Cd expression to suppress apoptosis in dopaminergic neurons by reducing p300 histone acetyltransferase activity. J Neurosci 31:2035-2051.

Kim HJ, Yun J, Lee J, Hong H, Jeong J, Kim E, Bae YS, Lee KJ (2011a) SUMO1 attenuates stress-induced ROS generation by inhibiting NADPH oxidase 2. Biochem Biophys Res Commun 410:555-562.

Kim YM, Jang WH, Quezado MM, Oh Y, Chung KC, Junn E, Mouradian MM (2011b) Proteasome inhibition induces $\alpha$-synuclein SUMOylation and aggregate formation. J Neurol Sci 307:157-161.

Kleinknecht A, Popova B, Lázaro DF, Pinho R, Valerius O, Outeiro TF, Braus GH (2016) C-terminal tyrosine residue modifications modulate the protective phosphorylation of serine 129 of $\alpha$-synuclein in a yeast model of Parkinson's disease. PLoS Genet 12: e1006098.

Krumova P, Meulmeester E, Garrido M, Tirard M, Hsiao HH, Bossis $G$, Urlaub $H$, Zweckstetter $M$, Kügler $S$, Melchior $F$, Bähr $M$, Weishaupt JH (2011) Sumoylation inhibits $\alpha$-synuclein aggregation and toxicity. J Cell Biol 194:49-60.

Lazzara CL, Riley RR, Rane A, Andersen JK, Kim YH (2015) The combination of lithium and L-dopa/carbidopa reduces MPTP-induced abnormal involuntary movements (AIMs) in mice: relevance for Parkinson's disease therapy. Brain Res 1622:127-136.

Lee YJ, Mou Y, Maric D, Klimanis D, Auh S, Hallenbeck JM (2011) Elevated global SUMOylation in Ubc9 transgenic mice protects their brains against focal cerebral ischemic damage. PLoS one 6: e25852.

Lee YJ, Mou Y, Klimanis D, Bernstock JD, Hallenbeck JM (2014) Global SUMOylation is a molecular mechanism underlying hypothermia-induced ischemic tolerance. Front Cell Neurosci 4:416.

Liebelt F, Vertegaal AC (2016) Ubiquitin-dependent and independent roles of SUMO in proteostasis. Am J Physiol Cell Physiol 311: C284-C296.
Lu S, Sung T, Lin N, Abraham RT, Jessen BA (2017) Lysosomal adaptation: how cells respond to lysosomotropic compounds. PLoS One 12:e0173771.

Marblestone JG, Edavettal SC, Lim Y, Lim P, Zuo X, Butt TR (2006) Comparison of SUMO fusion technology with traditional gene fusion systems: enhanced expression and solubility with SUMO. Protein Sci 15:182-189.

Martinez-Vicente M, Vila M (2013) Alpha-synuclein and protein degradation pathways in Parkinson's disease: a pathological feedback loop. Exp Neurol 247:308-313.

Pan T, Kondo S, Le W, Jankovic J (2008) The role of autophagy-lysosome pathway in neurodegeneration associated with Parkinson's disease. Brain 131:1969-1978.

Pandey D, Chen F, Patel A, Wang CY, Dimitropoulou C, Patel VS, Rudic RD, Stepp DW, Fulton DJ (2011) SUMO1 negatively regulates reactive oxygen species production from NADPH oxidases. Arterioscler Thromb Vasc Biol 31:1634-1642.

Peng X, Peng XM, Tehranian R, Dietrich P, Stefanis L, Perez RG (2005) Alpha-synuclein activation of protein phosphatase 2A reduces tyrosine hydroxylase phosphorylation in dopaminergic cells. J Cell Sci 118:3523-3530.

Polinski NK, Volpicelli-Daley LA, Sortwell CE, Luk KC, Cremades N, Gottler LM, Froula J, Duffy MF, Lee VMY, Martinez TN, Dave KD (2018) Best practices for generating and using $\alpha$-synuclein preformed fibrils to model Parkinson's disease in rodents. J Parkinsons Dis 8:303-322.

Popova B, Kleinknecht A, Braus GH (2015) Posttranslational modifications and clearing of $\alpha$-synuclein aggregates in yeast. Biomolecules 5:617-634.

Pranke IM, Morello V, Bigay J, Gibson K, Verbavatz JM, Antonny B, Jackson CL (2011) $\alpha$-Synuclein and ALPS motifs are membrane curvature sensors whose contrasting chemistry mediates selective vesicle binding. J Cell Biol 194:89-103.

Rodriguez MS, Dargemont C, Hay RT (2001) SUMO-1 conjugation in vivo requires both a consensus modification motif and nuclear targeting. J Biol Chem 276:12654-12659.

Rott R, Szargel R, Shani V, Hamza H, Savyon M, Abd Elghani F, Bandopadhyay R, Engelender S (2017) SUMOylation and ubiquitination reciprocally regulate $\alpha$-synuclein degradation and pathological aggregation. Proc Natl Acad Sci USA 114:13176-13181.

Sampson DA, Wang M, Matunis MJ (2001) The small ubiquitin-like modifier-1 (SUMO-1) consensus sequence mediates Ubc9 binding and is essential for SUMO-1 modification. J Biol Chem 276:21664-21669.

Scott D, Roy S (2012) $\alpha$-synuclein inhibits intersynaptic vesicle mobility and maintains recycling-pool homeostasis. J Neurosci 32:10129-10135.

Shahpasandzadeh H, Popova B, Kleinknecht A, Fraser PE, Outeiro TF, Braus GH (2014) Interplay between sumoylation and phosphorylation for protection against $\alpha$-synuclein inclusions. J Biol Chem 289:31224-31240.

Shimura H, Schlossmacher MG, Hattori N, Frosch MP, Trockenbacher A, Schneider R, Mizuno Y, Kosik KS, Selkoe DJ (2001) Ubiquitination of a new form of $\alpha$-synuclein by parkin from human brain: implications for Parkinson's disease. Science 293:263-269.

Siderowf A, Stern M (2003) Update on Parkinson's disease. Ann Intern Med 138:651-658.

Spillantini MG, Schmidt ML, Lee VM, Trojanowski JQ, Jakes R, Goedert M (1997) Alpha-synuclein in Lewy bodies. Nature 388:839-840.

Sun H, Lu L, Zuo Y, Wang Y, Jiao Y, Zeng WZ, Huang C, Zhu MX, Zamponi GW, Zhou T, Xu TL, Cheng J, Li Y (2014) Kainate receptor activation induces glycine receptor endocytosis through PKC deSUMOylation. Nat Commun 5:4980.

Volpicelli-Daley LA, Luk KC, Patel TP, Tanik SA, Riddle DM, Stieber A, Meaney DF, Trojanowski JQ, Lee VM (2011) Exogenous $\alpha$-synuclein fibrils induce Lewy body pathology leading to synaptic dysfunction and neuron death. Neuron 72:57-71. 
Vijayakumaran S, Wong MB, Antony H, Pountney DL (2015) Direct and/or indirect roles for SUMO in modulating $\alpha$-synuclein toxicity. Biomolecules 5:1697-1716.

Webb JL, Ravikumar B, Atkins J, Skepper JN, Rubinsztein DC (2003) Alpha-synuclein is degraded by both autophagy and the proteasome. J Biol Chem 278:25009-25013.

Wilkinson KA, Henley JM (2010) Mechanisms, regulation and consequences of protein SUMOylation. Biochem J 428:133-145.

Yan FF, Lin CW, Cartier EA, Shyng SL (2005) Role of ubiquitin-proteasome degradation pathway in biogenesis efficiency of [beta]cell ATP-sensitive potassium channels. Am J Physiol Cell Physiol 289:C1351-C1359.
Zhou P, Chen X, Li M, Tan J, Zhang Y, Yuan W, Zhou J, Wang G (2019) 2-D08 as a SUMOylation inhibitor induced ROS accumulation mediates apoptosis of acute myeloid leukemia cells possibly through the deSUMOylation of NOX2. Biochem Biophys Res Commun 513:1063-1069.

Zhu M, Qin ZJ, Hu D, Munishkina LA, Fink AL (2006) Alpha-synuclein can function as an antioxidant preventing oxidation of unsaturated lipid in vesicles. Biochemistry 45:8135-8142.

Zhu LN, Qiao HH, Chen L, Sun LP, Hui JL, Lian YL, Xie WB, Ding JY, Meng YL, Zhu BF, Qiu PM (2018) SUMOylation of $\alpha$-synuclein influences on $\alpha$-synuclein aggregation induced by methamphetamine. Front Cell Neurosci 12:262. 